\title{
Die psychiatrische Begutachtung von Kindern mit «abnormen Reaktionen» in der Zürcher Kinderbeobachtungsstation Brüschhalde 1957 bis 1972
}

\author{
Galle, Sara ; Neuhaus, Emmanuel ; Künzle, Lena ; Lis, Daniel ; Ritzmann, Iris
}

\begin{abstract}
The paper deals with the psychiatric assessment of personality in connection with the out-ofhome-placement of children. The focus lies on the assessment of two children, who were both born out of wedlock and admitted by their guardians to the psychiatric child observation ward Brüschhalde in the Canton of Zurich in 1957 and 1972. The authors show how personality was conceptualised, recorded and evaluated in an in-patient context. The doctors examining the two children diagnosed «abnormal», or more precisely «neurotic reactions», which were triggered by «unfavourable social conditions» and led to developmental disorders. This was in fact the most common diagnosis at the Brüschhalde in those years. The personality concept of child psychiatry represented a developmental concept which aimed not only at personal development but also at social assimilation. The most important prerequisite for a «normal» personality to develop, was and remained a «decent, harmonious family life». Out-of-home- placement of children was therefore not mainly based on the diagnoses, but on the evaluation of family background and the personality of the parents, especially the mothers.
\end{abstract}

DOI: https://doi.org/10.24894/Gesn-de.2020.77010

Posted at the Zurich Open Repository and Archive, University of Zurich

ZORA URL: https://doi.org/10.5167/uzh-194593

Journal Article

Published Version

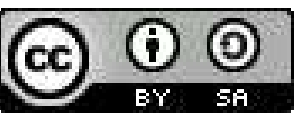

The following work is licensed under a Creative Commons: Attribution-ShareAlike 4.0 International (CC BY-SA 4.0) License.

Originally published at:

Galle, Sara; Neuhaus, Emmanuel; Künzle, Lena; Lis, Daniel; Ritzmann, Iris (2020). Die psychiatrische Begutachtung von Kindern mit «abnormen Reaktionen» in der Zürcher Kinderbeobachtungsstation Brüschhalde 1957 bis 1972. Gesnerus, 77(2):206-243.

DOI: https://doi.org/10.24894/Gesn-de.2020.77010 


\title{
Die psychiatrische Begutachtung von Kindern mit «abnormen Reaktionen» in der Zürcher Kinder- beobachtungsstation Brüschhalde 1957 bis 1972
}

Sara Galle, Emmanuel Neuhaus, Lena Künzle, Daniel Lis, Iris Ritzmann

\begin{abstract}
Der Beitrag setzt sich mit der psychiatrischen Beurteilung der Persönlichkeit im Zusammenhang mit der Fremdplatzierung von Kindern auseinander. Im Zentrum steht die Begutachtung zweier Kinder, die 1957 beziehungsweise 1972 von ihren Vormunden in die kinderpsychiatrische Beobachtungsstation Brüschhalde im Kanton Zürich eingewiesen wurden. Die Autorinnen und Autoren zeigen auf, wie Persönlichkeit im stationären Kontext konzeptualisiert, erfasst und bewertet wurde. Bei den beiden ausserehelich geborenen Kindern diagnostizierten die begutachtenden Ärztinnen und Ärzte, wie bei den meisten Kindern in der Brüschhalde zu jener Zeit, «abnorme», genauer «neurotische Reaktionen», die durch «ungünstige Milieuverhältnisse» ausgelöst worden waren und zu Entwicklungsstörungen führten.

Das Persönlichkeitskonzept der Kinderpsychiatrie stellte ein Entwicklungskonzept dar, das nicht nur die individuelle Entfaltung, sondern auch die gesellschaftliche Assimilation zum Ziel hatte. Die wichtigste Voraussetzung für eine «normale» Persönlichkeitsentwicklung des Kindes war und blieb ein «geordnetes, harmonisches Familienleben». Massgeblich für die Fremdplatzierung war nicht die Diagnose, sondern die familiäre Konstellation und die Persönlichkeit der Eltern, insbesondere der Mütter.

Psychiatrische Expertise, Fremdplatzierung, Kinder- und Jugendfürsorge, soziale Integration und Exklusion
\end{abstract}

Dr. Sara Galle, Fachhochschule Nordwestschweiz, Hochschule für Soziale Arbeit, Institut Integration und Partizipation, Riggenbachstrasse 16, 4600 Olten, sara.galle@fhnw.ch, +41629572304. 


\section{Psychiatric assessment of children with «abnormal reactions». The examination practice at the Zurich children's observation ward Brüschhalde 1957 to 1972}

The paper deals with the psychiatric assessment of personality in connection with the out-of-home-placement of children. The focus lies on the assessment of two children, who were both born out of wedlock and admitted by their guardians to the psychiatric child observation ward Brüschhalde in the Canton of Zurich in 1957 and 1972. The authors show how personality was conceptualised, recorded and evaluated in an in-patient context. The doctors examining the two children diagnosed «abnormal», or more precisely «neurotic reactions», which were triggered by «unfavourable social conditions» and led to developmental disorders. This was in fact the most common diagnosis at the Brüschhalde in those years.

The personality concept of child psychiatry represented a developmental concept which aimed not only at personal development but also at social assimilation. The most important prerequisite for a «normal» personality to develop, was and remained a «decent, harmonious family life». Out-ofhome-placement of children was therefore not mainly based on the diagnoses, but on the evaluation of family background and the personality of the parents, especially the mothers.

Psychiatric Expertise, Child Placement, Child Welfare, Social Integration and Social Exclusion

«Wie beurteilen Sie [die] Persönlichkeit [des Kindes] im gegenwärtigen Zeitpunkt?» $\gg^{1}$ Mit dieser Frage wandten sich Zürcher Amtsvormunde an die Leitung der kinderpsychiatrischen Beobachtungsstation Brüschhalde, wenn sie diese mit der Begutachtung ihrer Mündel beauftragten. Sie war stets verbunden mit behördlichen Erwägungen zur Fremdplatzierung der Kinder. Ausgehend von den Fallbeispielen zweier Kinder, die von ihren Vormunden in die Brüschhalde eingewiesen und bei denen «neurotische Reaktionen» diagnostiziert wurden, zeigen wir auf, wie Persönlichkeit im Kontext der stationären Kinderpsychiatrie konzeptualisiert, erfasst und bewertet wurde. Dabei interessiert uns insbesondere, wie die Konstruktion abweichenden Verhaltens mit gesellschaftlichen Normen korrespondierte.

1 Staatsarchiv des Kantons Zürich (StAZH), Z 98, Dossier Nr. 3483, Schreiben des Amtsvormunds an das Kantonale Kinderheim Brüschhalde vom 23.02.1972. 
Fremdplatzierungen von Kindern und Jugendlichen sind in jüngster Zeit international vermehrt Gegenstand der historischen Forschung geworden. ${ }^{2}$ In der Schweiz haben sich bisher einzelne Studien und ein grösseres, vom Schweizerischen Nationalfonds gefördertes Forschungsprojekt vor allem mit der Rolle der Behörden und privater Organisationen bei der Wegnahme der Kinder aus ihren Familien und deren Platzierung in Pflegefamilien, Kinderheimen und Erziehungsanstalten beschäftigt. ${ }^{3}$ Bei anderen standen die Auswirkungen der behördlichen Massnahmen und der ausserfamiliären Erziehung auf die Biografien der betroffenen Personen im Zentrum oder sie untersuchten Formen von Gewalt und Missbrauch in Heimen. ${ }^{4}$ Noch kaum untersucht ist die Rolle kinderpsychiatrischer Expertise bei der Fremdplatzierung von Kindern und Jugendlichen. ${ }^{5}$

Die folgenden Ausführungen basieren auf einem Forschungsprojekt, das sich mit der Genese von kinderpsychiatrischen Gutachten und ihrer Auswirkung auf die Identitätskonstruktionen der betroffenen Personen befasst. ${ }^{6}$

2 Eine Zusammenstellung der zumeist im Auftrag von Regierungen veranlassten Untersuchungen zum institutionellen Missbrauch von Kindern findet sich auf http://www.lib.latrobe. edu.au/research/ageofinquiry/index.html. Zu den rechtlichen Grundlagen der historischen Aufarbeitung in der Schweiz vgl. www.bj.admin.ch/bj/de/home/gesellschaft/fszm.html (Zugriff am 6.2.2020).

3 Zum Sinergia-Projekt «Placing Children in Care. Child welfare in Switzerland 1940-1990» vgl. www.placing-children-in-care.ch/ (Zugriff am 6.2.2020).

4 Vgl. dazu insbes. Lengwiler et al. 2013, Hauss et al. 2018 und Furrer et al. 2014. Berichte zu Heimen liegen etwa vor im Kanton Luzern, Thurgau und Zürich: Akermann et al. 2012 und 2015, Ries/Beck 2013 und Bombach et al. 2017. Zur Anstaltslandschaft im Kanton Zürich vgl. Jenzer/Meier 2018.

5 Zur Rolle der psychiatrischen Begutachtung bei der Fremdplatzierung von Kindern und Jugendlichen vgl. Businger/Ramsauer 2019, Janett 2018 und Galle 2016; in Strafprozessen vgl. Germann 2004 und 2017; im Zusammenhang mit Sterilisationen vgl. insbes. Dubach 2013 und Hauss/Ziegler 2009; im Rahmen des Sozialversicherungsrechts vgl. Lengwiler 2017; bei behördlichen Zwangseinweisungen von Jugendlichen und Erwachsenen in geschlossene Anstalten und Kliniken vgl. Bühler et al. 2019. Zur Geschichte der Psychiatrie im Kanton Zürich vgl. Meier et al. 2007. Zur stationären psychiatrischen Begutachtung von Kindern in Österreich vgl. Dietrich-Daum et al. 2019, Dietrich-Daum/Ralser 2018, Hähner-Rombach 2017 und Ralser 2017.

6 Das Forschungsprojekt ist Teil des 2018 gestarteten und auf insgesamt fünf Jahre angelegten Nationalen Forschungsprogramms (NFP 76) zu «Fürsorge und Zwang», in dem sich 27 Projekte mit den Merkmalen, Mechanismen und Wirkungsweisen der schweizerischen Fürsorgepolitik und -praxis in verschiedenen Kontexten beschäftigen. Zum von Iris Ritzmann geleiteten und vom Schweizerischen Nationalfonds geförderten Forschungsprojekt vgl. http:// www.nfp76.ch/de/projekte/rechte-und-rechtspraxis/projekt-ritzmann. Zum NFP 76 vgl. http://www.nfp76.ch/de (Zugriff am 6.2.2020). 


\section{Analyse der Begutachtung zweier Kinder in den Jahren 1957 und 1972}

Grundlage des vorliegenden Beitrags bilden die Fallakten zweier Kinder, die 1957 und 1972 in der kinderpsychiatrischen Beobachtungsstation Brüschhalde begutachtet wurden. Die 1921 als Aussenstation der Heil- und Pflegeanstalt Burghölzli in der Stephansburg in Zürich eingerichtete Beobachtungsstation zählt zu den frühesten kinderpsychiatrischen Einrichtungen im deutschsprachigen Raum.7 Sie war die erste ihrer Art in der Schweiz und wurde nicht zufällig in Zürich gegründet. Dieser Umstand war nicht nur der in der Deutschschweiz führenden Rolle der psychiatrischen Universitätsklinik Burghölzli geschuldet, sondern auch der vergleichsweise früh einsetzenden Institutionalisierung und Professionalisierung der Kinder- und Jugendfürsorge in der Stadt und im Kanton. ${ }^{8}$ Mit der 1944 erfolgten Übersiedlung ins ehemalige Waisenhaus Brüschhalde in Männedorf, eine Gemeinde am Zürichsee, wurde die räumliche Trennung von der Erwachsenenpsychiatrie noch deutlicher vollzogen. ${ }^{9}$ Die Brüschhalde blieb im untersuchten Zeitraum die einzige stationäre kinder- und jugendpsychiatrische Einrichtung im Kanton Zürich. ${ }^{10}$

Die Fürsorge interessierte sich für die Psychiatrie, weil es aus Sicht der Behörden mit den psychiatrischen Diagnosen und Prognosen zum Krankheitsverlauf möglich wurde, das künftige Verhalten der von ihnen betreuten Personen zu antizipieren, was die Prävention immer wichtiger und die Erfassung der Persönlichkeit zum zentralen Anliegen machte. Dies zeigt sich auch im Selbstverständnis der sich im 20. Jahrhundert formierenden Kinderpsychiatrie. So galten die «Prophylaxe und Psychohygiene» als deren wichtigste Aufgabe, wozu die Früherfassung sogenannter Risikogruppen gehörte. ${ }^{11}$ Seit 1929 leitete der Kinderpsychiater Jakob Lutz (1903-1998) die Beobachtungsstation. Er stand zugleich dem 1947 geschaffenen Kinderpsychiatrischen Dienst des Kantons Zürich vor, lehrte ab 1949 als Titular- und später als ausserordentlicher Professor für Kinderpsychiatrie an der Universität Zürich und verfasste mehrere auch international anerkannte Schriften. ${ }^{12}$

Der Fokus auf das Jahr 1957 ist forschungspragmatisch begründet. Das Gutachten über Anna, wie wir sie hier nennen, war das erste, das uns im Kon-

7 Dietrich-Daum/Ralser 2018, 113.

8 Zürrer-Simmen 1994, 45-57.

9 Zürrer-Simmen 1994, 8, 83.

10 Vgl. Jenzer/Meier 2018, 97.

11 Lutz 1969a, 105; Herzka 1973, 140. Vgl. auch Lutz 1929, 13-14, sowie Lutz 1935, 16.

12 Lutz 1961; Steinhausen 2008. Der Kinderpsychiatrische Dienst umfasste neben der Beobachtungsstation Brüschhalde die kinderpsychiatrische Poliklinik in Zürich, die poliklinischen Zweig- und Regionalstellen in den Bezirken und später die Tagesklinik. 
text der Fallakte zugänglich war. ${ }^{13}$ In den darauffolgenden Jahren erfolgte eine Phase des gesellschaftlichen und institutionellen Wandels. Das Gutachten von 1972 haben wir so ausgewählt, dass die Effekte dieses Wandels in der kinderpsychiatrischen Begutachtung ersichtlich werden können. Das zentrale Kriterium, das die Wahl des zweiten Gutachtens bestimmte, war die Diagnose. Bei beiden Kindern war in der Brüschhalde eine «abnorme», genauer eine «neurotische Reaktion» diagnostiziert worden. Bereits 1957 waren die «neurotischen Reaktionen» und «neurotischen Persönlichkeitsentwicklungen» mit knapp 40 Prozent die häufigste Diagnose in der Brüschhalde. Bis 1972 stieg deren Anteil gar auf annährend 90 Prozent an. ${ }^{14}$ Das entsprach einem längerfristigen Trend, wie ihn der Schweizer Kinderpsychiater Moritz Tramer (1882-1963) bereits 1949 beschrieben hatte. ${ }^{15}$ Dies obwohl sowohl die Ursachen als auch der Status der Diagnose als Krankheit umstritten blieben. ${ }^{16}$ Das Fehlen einer einheitlichen Definition ermöglichte der Kinderpsychiatrie, verschiedenste soziale Problemlagen auf der Ebene des «neurotischen» Individuums zu beurteilen. ${ }^{17}$

Im Fokus der Kinderpsychiatrie stand die Familie, allen voran die Eltern, sowie das soziale Umfeld der Kinder. Wie unsere Auswertungen zeigen, kamen mehr als zwei Drittel der Kinder, bei denen 1972 in der Brüschhalde eine «neurotische Reaktion» diagnostiziert wurde, aus sogenannt unvollständigen Familien, deren Eltern entweder unverheiratet, getrennt, geschieden, verwitwet, verstorben oder abwesend waren..$^{18}$ Die den folgenden Ausführungen zugrundliegenden Gutachten betreffen Kinder, die ausserehelich geboren wurden, bevormundet und in einer Pflegefamilie platziert waren, wo die von den Vormunden beschriebenen «Schwierigkeiten» auftraten.

Bei der Beratung des Gesetzesentwurfs zum Schweizerischen Zivilgesetzbuch von 1907, das 1912 in Kraft trat, war eine Mehrheit der Ansicht,

13 Dem Forschungsprojekt wurde erst im April 2019, neun Monate nach Beginn im Juli 2018, uneingeschränkter Zugang zu den Fallakten im Staatsarchiv Zürich gewährt. Die Fallakte von Anna haben wir als Kopie von der heute 70-jährigen Frau erhalten, mit der wir auch mehrere Interviews führen durften. $\mathrm{Zu}$ den ethischen Anforderungen an die Forschungsprojekte des NFP76, insbesondere zum Persönlichkeits- und Datenschutz vgl. http://www. nfp76.ch/SiteCollectionDocuments/nfp76-ethische-anforderungen-an-die-forschungsprojekted.pdf (Zugriff am 6.2.2020)

14 Diese Angaben beruhen auf einer vollständigen Erhebung der Fallakten der beiden Jahre 1957 und 1972. Andernorts, z.B. in der Innsbrucker Kinderbeobachtungsstation, zählten dagegen «organische Störungen und Einschränkungen (Oligophrenien, Epilepsien und Hirnschädigungen)» zu den häufigsten Diagnosen. Vgl. Hähner-Rombach 2017, 330.

15 Tramer 1949, 19. Vgl. zu Tramer Jorisch-Wissink 1986.

16 Hoffmann 1986, 30.

17 Kochmann 1963, 23-24; Canziani 1995, 85. Dass die Legitimierung der Psychiatrie als Expertin in sozialen Fragen bereits in den Krankheitsbildern angelegt ist, zeigt Brigitta Bernet. Vgl. Bernet 2006.

18 Eigene Erhebungen (Anm. 14). 
dass das Kind zahlreichen körperlichen und moralischen Gefahren ausgesetzt sei, wenn es nicht in geordneten Familienverhältnissen aufwachse. ${ }^{19}$ Es setzte sich im Parlament das bürgerliche Familienideal durch, das dem Mann die Rolle des Ernährers und Familienoberhaupts zuwies und die Bestimmung der Frau als Mutter, Ehegattin und Hausfrau festschrieb. ${ }^{20}$ Kinder unverheirateter Mütter erhielten in der Schweiz bis zur Revision des Familienrechts 1976, die 1978 in Kraft trat, von Rechts wegen einen Beistand. Nach Ermittlung der Vaterschaft wurde die Beistandschaft in der Regel in eine Vormundschaft umgewandelt. ${ }^{21}$ Die Mutter und der Vater, sofern er das Kind als sein eigenes anerkannt hatte, konnten die Obhut und die «elterliche Gewalt» (heute: Sorgerecht) für das Kind beantragen. Dafür mussten sie aber Gewähr bieten, dass das Kindeswohl nicht gefährdet war. ${ }^{22}$ Wie die Untersuchung der Rechtspraxis zeigt, wurden bis zur Revision der familienrechtlichen Bestimmungen 1976 viele ausserehelich geborene Kinder fremdplatziert und nur selten erhielten ledige Mütter die elterliche Gewalt für ihre Kinder. ${ }^{23}$

\section{Konzeption stationärer Begutachtung und Funktion psychiatrischer Expertisen im kantonalen Fürsorgedispositiv}

Die Brüschhalde verzeichnete seit den 1950er Jahren wesentliche institutionelle Veränderungen, die die Begutachtung beeinflussten. Mit der Eröffnung einer Therapiestation sowie Einrichtungen für Spieldiagnostik und -therapie wurde das Behandlungsangebot 1959 stark ausgebaut. ${ }^{24}$ Eine personelle Zäsur bedeutete der Rücktritt von Jakob Lutz als Direktor des kantonalen Kinderpsychiatrischen Dienstes 1968, nachdem dieser der Zürcher Kinderpsychiatrie während fast vierzig Jahren vorgestanden hatte. ${ }^{25} \mathrm{Zu} \mathrm{Lutz'Nachfol-}$ ger wurde Robert Jules Corboz (1919-1987) ernannt, der bereits seine Facharztausbildung in der Brüschhalde und der Poliklinik absolviert hatte und seit 1952 in den beiden Institutionen als Oberarzt tätig war. Mit diesem Entscheid setzten die politisch Verantwortlichen auf Kontinuität in der ins-

19 Tuor 1968, 234-235.

20 Ramsauer 2000,30-34.

21 Art. 311 Zivilgesetzbuch (ZGB).

22 Zum ausserehelichen Kindesverhältnis vgl. die Artikel 302-327 ZGB. Zum Kindeswohl vgl. das folgende Kapitel.

23 Vgl. Businger/Ramsauer 2019, 21-29; Galle 2016, 139-140.

24 Kinderpsychiatrischer Dienst des Kantons Zürich (KPD, später: Kinder- und Jugendpsychiatrischer Dienst, KJPD), Jahresbericht 1959, 1-2; ebd., Jahresbericht 1971, 2; Lutz 1969a, 102. Zur Spieltherapie als psychotherapeutisches Verfahren vgl. Lutz 1961, 311.

25 KPD, Jahresbericht 1968, 1. Vgl. auch Steinhausen 2008. 
titutionellen Leitung. ${ }^{26}$ Corboz führte auch die Lehrtätigkeit seines Vorgängers fort und wurde 1970 Ordinarius des neu geschaffenen Lehrstuhls für Kinder- und Jugendpsychiatrie an der Universität Zürich. Die Kinder- und Jugendpsychiatrie machte seitdem einen Viertel des Psychiatrieunterrichts im Medizinstudium aus, ${ }^{27}$ so dass die in der Brüschhalde tätigen Assistenzärztinnen und -ärzte über fundiertere Fachkenntnisse verfügten als zuvor.

Im untersuchten Zeitraum kam es überdies zu einer erheblichen Aufstockung und Diversifizierung des Personals, während die Zahl der aufgenommenen Kinder und der Plätze stabil blieben. ${ }^{28}$ Waren 1957 neben einer Assistenzärztin bloss ein Lehrer und vier Erzieherinnen in der Brüschhalde angestellt, umfasste das Personal 1972 einen zweiten Assistenzarzt, eine leitende Oberärztin, ferner eine Psychologin und einen Psychologen, zwei zusätzliche Lehrkräfte, drei psychotherapeutisch ausgebildete Mitarbeitende sowie eine Fürsorgerin, die für Abklärungen der familiären Verhältnisse und zur Beratung der Eltern zuständig war. ${ }^{29}$ Für die Ärztinnen und Ärzte blieb folglich trotz der vollen Auslastung der Beobachtungsstation insgesamt mehr Zeit für die Begutachtung der einzelnen Kinder, was sich auch im Umfang der Gutachten niederschlug.

Innerhalb des kantonalen Dispositivs der Kinderfürsorge hatte die Brüschhalde die Funktion einer Schnittstelle inne. Das zeigt sich am breiten Geflecht der einweisenden Instanzen, zu denen neben Vormundschaftsbehörden Jugend- und Fürsorgeämter, Jugendsekretariate, Jugendanwaltschaften und Bezirksgerichte, schulpsychologische und -psychiatrische Dienste, Schulpflege und -ämter, Privatärztinnen und -ärzte, das Kinderspital sowie die kinderpsychiatrische Poliklinik und vereinzelt auch die Eltern zählten. ${ }^{30}$ Einweisungen von Behörden mit vormundschaftlichen und/oder jugendfürsorgerischen Aufgaben waren im untersuchten Zeitraum am häufigsten. ${ }^{31}$ In der Brüschhalde wurden laut den Jahresberichten jene Kinder bis 14 Jahre begutachtet, «deren Verhalten von Erziehern und Fürsorgern

26 StAZH, MM 3.123 RRB 1968/2933, Kinderpsychiatrischer Dienst (Personal), 25.07.1968, $1458-1459$.

27 KPD, Jahresbericht 1970, 1-2.

28 KPD, Jahresberichte 1957-1972.

29 Zum Personal zählte zudem der sogenannte Hausvater, der als Heimleiter fungierte, sowie die Verwaltungsanstellten. Vgl. KPD, Jahresbericht 1957, 2, und Jahresbericht 1972, 3. Eine ähnliche Entwicklung zeigt sich in der Kinderbeobachtungsstation in Innsbruck, vgl. Hähner-Rombach 2017, 321.

30 KPD, Jahresbericht 1957, 3, und Jahresbericht 1972, 4.

311957 wurden knapp zwei Drittel der Kinder von Behörden der Vormundschaft und Jugendfürsorge in die Brüschhalde eingewiesen und 1972 nahezu die Hälfte. Vgl. hierzu KPD, Jahresbericht 1957, 3, und Jahresbericht 1972, 4. Einweisungen in die Innsbrucker Beobachtungsstation wurden dagegen erstaunlicherweise viel häufiger von den Eltern oder andere Familienangehörige initiiert, vgl. Hähner-Rombach 2017, 323-324. 
nicht ohne weiteres verstanden werden kann». Die Poliklinik überwies Kinder, die «so schwer zu durchschauen [sind], dass eine klinische Beobachtung notwendig ist». ${ }^{32}$ In der Darstellung von Jakob Lutz hatte die Brüschhalde «den Charakter einer Zentrale für einen grossen Teil der Fragen, die die geistig abnormen Kinder und Jugendlichen betreffen». ${ }^{33}$ Die Angaben, welche Kinder begutachtet werden, waren wenig präzise, was der Kinderpsychiatrie ein grosses Tätigkeitfeld verschaffte.

Für die Vormunde bedeutete die Einweisung der von ihnen betreuten Kinder in eine stationäre Einrichtung immer auch eine entlastende Massnahme für akute Schwierigkeiten, wie die beiden Fallbeispiele zeigen werden. Die Vormundschaftsbehörden konnten bei «pflichtwidrigem Verhalten» der Eltern zum Schutz des Kindeswohls eine vorübergehende Wegnahme des Kindes anordnen. ${ }^{34}$ Eine Fremdplatzierung mussten sie zwingend veranlassen, wenn das «geistige oder leibliche Wohl» des Kindes «dauernd gefährdet» oder das Kind «verwahrlost» war. ${ }^{35}$ Wann Behörden und Vormunde eine psychiatrische Begutachtung beantragten, lag gänzlich in ihrem Ermessen und war gesetzlich nicht vorgeschrieben. Vormunde erwogen für ihre minderjährigen Mündel häufig dann eine stationäre Begutachtung, wenn diese die Ordnung in Kinderheimen und Erziehungsanstalten «störten» oder wenn wie in den beiden Fallbeispielen «Schwierigkeiten» in der Herkunfts- oder Pflegefamilie entstanden. ${ }^{36}$ Von den 1957 und 1972 in die Brüschhalde eingewiesenen Kindern war insgesamt knapp ein Drittel bereits vorher fremdplatziert. ${ }^{37}$ Häufig und im untersuchten Zeitraum zunehmend wurden Kinder stationär begutachtet, wenn sie wie erwähnt aus sogenannt ungeordneten Familienverhältnissen kamen. Die Vorstellung der bürgerlichen Familie als ideales Umfeld für ein gesundes Aufwachsen von Kindern büsste nicht an Bedeutung ein.

\section{Familie, Persönlichkeit und Kindeswohl in der kinderpsychiatrischen Begutachtung}

Für den deutsch-jüdischen Kinderpsychiater Erich Benjamin (1880-1943) waren es die durch die «gegenwärtigen Lebensbedingungen» entstandenen «Schäden», die der «Einführung des Kindes in die Gemeinschaft im Wege

32 KPD, Jahresbericht 1969, 1.

33 Lutz 1935, 11.

34 Art. 283 ZGB.

35 Art. 284 ZGB.

36 Businger/Ramsauer 2019, 63; Galle 2016, 571. Zu Österreich vgl. Ralser 2017, 562-563.

37 Eigene Erhebung (Anm. 14), da diese Zahlen in den Jahresberichten nicht ausgewiesen werden. 
stehen», was die «Erziehung des Kindes heute mehr als je zu einem Problem» mache. $\mathrm{Zu}$ diesen «gegenwärtigen Lebensbedingungen» zählte Benjamin namentlich die Auflösung des Familienlebens sowie die Emanzipation der Frau.$^{38}$ Das Lehrbuch der Psychopathologie des Kindesalters für Ärzte und Erzieher von 1938, an dem neben Erich Benjamin, der kurz vor Erscheinen des Buches in die USA geflüchtet war, auch Jakob Lutz mitwirkte, galt in der Deutschschweiz für mehr als zwei Jahrzehnte als Standardwerk. In seinem 1961 erschienenen Lehrbuch für Kinderpsychiatrie, das für die ärztliche Ausbildung und Praxis in Zürich massgebend wurde, erwähnte Lutz als umweltbedingte «Störfaktoren» der kindlichen Entwicklung an erster Stelle die aussereheliche Geburt und die unvollständige Familie. Gleich an zweiter Stelle folgt die Persönlichkeit der Mutter. ${ }^{39}$ Lutz referierte hierbei auf seinen langjährigen Wegbegleiter, den renommierten Zürcher Heilpädagogen Heinrich Hanselmann (1885-1960), und befürwortete dessen Annahme, «dass alles, was mit Kontakt, Beziehung, Anschluss an die Umwelt zu tun habe, von der Mutter ausgehe und durch ihr Fehlverhalten entsprechend gefährdet sei».40 Die in der Psychologie immer stärker hervorgehobene Wichtigkeit der (emotionalen) Bindung zwischen Mutter und Kind hielt ab den 1960er und insbesondere in den 1970er Jahren auch verstärkt Einzug in die behördliche Praxis. ${ }^{41}$

Für Lutz stellte sich darüber hinausgehend die Frage, ob es sich bei der zunehmenden «Berufsarbeit» der Frauen und der dadurch eingebüssten «Mütterlichkeit» nicht gar um ein «Zivilisationsproblem» handle. ${ }^{42}$ Er sah im «ganz offensichtlich zunehmenden Verlust der Erziehungsfähigkeit der Eltern» die «gesunde Entwicklung» der Kinder gefährdet. ${ }^{43}$ Die von den Kinderpsychiatern wiederholt konstatierten Erziehungsschwierigkeiten waren aus seiner Sicht Ausdruck eines sozialen Wandels, der die natürliche Ordnung ins Wanken brachte, die Familie als Fundament des Staates erodieren liess und somit letztlich die hierarchisch-patriarchale Gesellschaftsordnung gefährdete. Die «Vernachlässigung» der Erziehung durch die Eltern war auch der häufigste Grund für die Anordnung von Kindesschutzschutzmassnahmen durch die Zürcher Behörden, insbesondere bei jüngeren Kindern. ${ }^{44}$ Ledige und geschiedene Mütter waren neben ärmeren Familien und ihren Kindern im untersuchten Zeitraum am häufigsten von diesen Massnahmen betroffen. ${ }^{45}$

38 Benjamin 1938, 152. Zum Leben und Wirken von Erich Benjamin vgl. Oechsle 2006.

39 Lutz 1961, 48, 331.

40 Lutz 1961, 50. Zum Vorhaben einer gemeinsamen Publikation vgl. das Vorwort, 9. Zu Hanselmann vgl. Heese 2017.

41 Businger/Ramsauer 2019, 35; Lutz 1961, 331.

42 Lutz 1957, 14.

43 Lutz 1969a, 105; Lutz 1984, 13.

44 Businger/Ramsauer 2019, 35.

45 Businger/Ramsauer 2019, 35, 120. 
Der Massstab für Massnahmen zum Schutz des Kindes war und ist aber sowohl in der Kinderpsychiatrie als auch in der gesetzlichen Grundlage formell das Kindeswohl. In den Rechtskommentaren und teilweise auch im Gesetz wird es synonym mit dem Kindesinteresse verwendet und dient als Ersatz für die fehlende Selbstbestimmung des Kindes. Es sollte einen sozialen Ausgleich für die schwächere Position des Kindes bilden ${ }^{46}$ Die Wahrung des Kindeswohls wurde mit der Revision der familienrechtlichen Bestimmungen von 1976 zur obersten Maxime erklärt. ${ }^{47}$ Der unbestimmte Rechtsbegriff erforderte indes stets eine Konkretisierung - eine Aufgabe, die die rechtsprechenden Behörden seit längerer Zeit zunehmend psychiatrischen Expertinnen und Experten überantworteten..$^{48}$

Die Frage nach der Beeinträchtigung des Kindeswohls und der Störung der kindlichen Entwicklung beantwortete die Kinderpsychiatrie mithilfe der Beobachtung und materialisierte sie in Gutachten. Jakob Lutz fasste die Beobachtungstätigkeit der Kinderpsychiatrie folgendermassen zusammen: «Die exakte körperliche und psychische ärztliche Untersuchung, die ärztliche klinische Beobachtung, ergänzt durch das, was Schul- und Erziehungsfachleute feststellen und das, was die Fürsorge an Milieubeobachtungen und Daten der Vorgeschichte beizutragen hat, sind die Elemente dessen, was wir unter psychiatrischer Kinderbeobachtung verstehen.» ${ }^{49}$ Den Assistenzärztinnen und -ärzten kam dabei eine zentrale Rolle zu. Sie führten nicht nur die Anamnese - das Ermitteln und Verfassen der «Vorgeschichte» der Patientinnen und Patienten - und die Exploration - die körperlichen und psychischen Untersuchungen und Beobachtungen im Heim - durch, sie waren auch für die Testverfahren verantwortlich und nahmen darüber hinaus therapeutische Funktionen wahr. Sie führten die Krankengeschichte und verfassten das Gutachten zuhanden der Auftrag gebenden Instanz.

Mit einem Gutachten ist ein ärztlicher Bericht gemeint, der in beiden Fallbeispielen im Auftrag einer Behörde erstellt wurde und das Begutachtungsverfahren abschloss. Der Fokus der vorliegenden Analyse liegt auf der Genese der Gutachten inklusive der Begutachtungslogiken. Dabei beschäftigen wir uns mit den Fragen, wer die Gutachten verfasste, auf Basis welcher Wissensformen und Fragestellungen sowie in welchen Handlungskontexten die Begutachtung erfolgte. Wie Alexa Geisthövel und Volker Hess betrachten wir Begutachtung als eine Tätigkeit, die eine Handlung vorbereitet. ${ }^{50}$ In

46 Brauchli 1982, 135-140.

47 Brauchli 1982, 164.

48 Brauchli 1982, 114-120; Businger/Ramsauer 2019, 26.

49 Lutz 1935, 9.

50 Geisthövel/Hess 2017, 13. 
den von uns untersuchten Fällen bildeten die Gutachten die Grundlage für einen behördlichen Entscheid. Das Gutachten sollte, wie es Jakob Lutz für die Krankengeschichte geltend gemacht hatte, «so folgerichtig sein wie eine gute Novelle». Die nötige Stringenz wird dabei durch Selektion, Zuspitzung und Ausschluss anderer Möglichkeiten erzielt: «Das Zusammenwirken der einzelnen Faktoren muss so zwingend und logisch erfasst und dargestellt sein, dass als ihre Folge nichts anderes als gerade die Störung möglich ist, die das Kind zu uns geführt hat.» ${ }^{51}$ Das bedeutet in anderen Worten, dass der Krankheitswert von als «abweichend» taxiertem Verhalten in einer «plausible[n] Geschichte» definiert wird. ${ }^{52}$

\section{Auftrag und Fragestellung der einweisenden Vormunde an die Begutachtenden}

Im Zentrum der folgenden Ausführungen stehen die Fallakten von Anna und Roland. Der Vormund der achtjährigen Anna reichte am 17. Januar 1957 den für eine Einweisung und Begutachtung in der Beobachtungsstation Brüschhalde notwendigen und begründeten Auftrag ein. ${ }^{53}$ Sein Mündel bereite «immer wieder erzieherische Schwierigkeiten an seinem Pflegeort», schrieb der Stadtzürcher Amtsvormund. Vor der «unumgänglichen Umplazierung [sic]» hielt er, wie bereits in früheren Fällen, eine Beobachtung in der Brüschhalde für dringend nötig.

In seinem Schreiben hielt er fest, dass die Versetzung seines Mündels an den Pflegeort in Bülach vorerst zu einer Beruhigung geführt habe. Doch bald hätten sich «auch dort die alten Fehler» gezeigt. Pflegerisch sei das Mädchen in der Familie sehr gut aufgehoben, der erzieherischen Aufgabe an diesem «schwierig gearteten, wohl z.T. [erblich] belasteten Kind» sei aber die «oft zu empfindsame Pflegemutter nicht mehr gewachsen». Auch in der Schule habe das Kind «trotz guter Intelligenz» gestört. Die Lehrerin, bei der Anna die zweite Klasse besuchte, hatte indes «keine Mühe» mit dem Kind. ${ }^{54}$

Auffallend ist die Fixierung des Vormundes auf negative Eigenschaften und fehlerhaftes Verhalten des Kindes. Selbst die offenbar positive Situation in der Schule erwähnte er unter umgekehrten Vorzeichen. Der Vormund

51 Lutz 1961, 62.

52 Germann 2017; Galle 2016, 624. Zum progredienten Verlauf in Krankengeschichten vgl. Meier 2015.

53 Aufnahmereglement des Kantonalen Kinderheims Brüschhalde Männedorf, o.D., in: StAZH, Z 98, Dossier Nr. 2422.

54 StAZH, Z 98, Dossier Nr. 2422, Schreiben des Amtsvormunds an die Leitung des Kantonalen Kinderheims Brüschhalde vom 17.1.1957, 1. 
war nicht nur überzeugt, dass es sich um ein «schwierig geartetes Kind» handelte, sondern er hatte auch bereits eine Erklärung dafür. Neben einer wahrscheinlichen erblichen Belastung des Kindes wies er die Adressaten seines Schreibens auf die vorangehende fehlerhafte Erziehung von Mutter und Grossmutter hin. Auf deren «Verwöhnung» habe das Kind mit «Trotz» und «Rollenspielen» reagiert. Der Vormund nahm die Deutung der Experten vorweg, die er wohl aus anderen ärztlichen Berichten kannte und geradezu Lehrbuchcharakter hatte, wie noch zu zeigen sein wird. Dazu gehören auch die Angaben über die Herkunftsfamilie des Kindes, die - wie der Amtsvormund wusste - für die ärztliche Begutachtung zentral waren. ${ }^{55}$ Die Mutter des ausserehelich geborenen Kindes, führte der Vormund aus, stamme selber aus «unglücklichen Verhältnissen», die Grosseltern seien geschieden, und auch die Mutter sei «schon in der Erziehung sehr schwierig gewesen». Sie habe ein «sprunghaftes Wesen», erscheine zeitweise häufig am Pflegeort des Kindes, wolle ihm mit Geschenken imponieren, um dann wieder monatelang zu verschwinden. Der Kindsvater solle ein «Staatenloser» sein, der in Amerika lebe, so genau wusste es der Amtsvormund nicht. Gewiss war nur: «Alimente sind uneinbringlich». Für die insgesamt drei ausserehelich geborenen Kinder von Annas Mutter kam das Fürsorgeamt in Verbindung mit den heimatlichen Behörden auf. ${ }^{56}$

Zwei Aspekte kommen hier zum Tragen, die für die Begutachtung Annas bestimmend waren: Erstens erscheinen die Erziehungsschwierigkeiten im Schreiben des Vormundes als eine familiäre Konstante und werden in Zusammenhang mit dem Wesen der Mutter gebracht. Zweitens wird der sozioökonomische Status der Familie problematisiert. Bei Anna handelte es sich nicht nur um ein ausserehelich geborenes Kind, sondern bereits die Eltern ihrer Mutter hatten sich scheiden lassen. Der Vormund wies auf die daraus entstandenen Kosten hin, die der Staat zu tragen hatte. Von der ärztlichen Begutachtung erhoffte sich der Amtsvormund ganz konkret, «eine Wegleitung für die bestmögliche Plazierung [sic] [...] zu bekommen». ${ }^{57}$ Der ärztliche Bericht sollte also handlungsleitend sein, aber in erster Linie die erneute Umplatzierung gegenüber der Vormundschaftsbehörde legitimieren. Nicht das Kind und sein Wohl standen im Zentrum des Auftrages, sondern wie mit einer möglichst passenden Platzierung verhindert werden konnte, dass die «Kinderfehler» für die Erziehungsberechtigten und den

55 Zur zunehmenden Bedeutung psychiatrischen Wissens in der vormundschaftlichen Behördenpraxis vgl. Businger/Ramsauer 2019, 38, 86, 195, und Janett 2018, 263-264.

56 StAZH, Z 98, Dossier Nr. 2422, Schreiben des Amtsvormunds an die Leitung des Kantonalen Kinderheims Brüschhalde vom 17.1.1957, 1.

57 StAZH, Z 98, Dossier Nr. 2422, Schreiben des Amtsvormunds an die Leitung des Kantonalen Kinderheims Brüschhalde vom 17.1.1957, 2. 
Vormund erneut zum Problem werden. Die Erziehungsschwierigkeiten und ihre Ursachen erscheinen im behördlichen Schreiben nicht als Frage, vielmehr als Feststellung und Ausgangspunkt für die ärztliche Gutachtertätigkeit. Sie waren aus Sicht des Vormunds familiär und vermutlich erblich bedingt. Die Verhältnisse in der Pflegefamilie, so werden die weiteren Ausführungen zeigen, waren aber aus psychiatrischer Perspektive ebenfalls problematisch. Warum dies bei der Begutachtung von Anna eine untergeordnete Rolle spielte, steht im engen Zusammenhang mit der Logik der ärztlichen Gutachtertätigkeit und den gesellschaftlichen Vorstellungen der damaligen Psychopathologie, die vor allem Kinder von rechtlich, sozial und ökonomisch benachteiligten Eltern zu «schwierigen» Kindern mit einer «abnormen Persönlichkeit» werden liessen..$^{58}$

Für den Vormund des ebenfalls ausserehelich geborenen Roland, der 15 Jahre später in der Brüschhalde begutachtet wurde, standen im Gutachterauftrag vom 23. Februar 1972 gleichsam Fragen zur Umplatzierung des damals sechsjährigen Knaben im Zentrum, der wie Anna in einer Pflegefamilie untergebracht war. Der Auftrag unterscheidet sich aber in verschiedener Hinsicht. Der Vormund hatte bereits eine Wegnahme und Platzierung seines Mündels bei dessen inzwischen verheirateten Mutter eingeleitet. Doch schon bei den «vereinbarten Besuchen des Kindes im vorgesehenen neuen Milieu» kam es zu «Schwierigkeiten». Daraufhin empfahl die Zweigstelle Winterthur des Kinderpsychiatrischen Dienstes nach einer ambulanten Untersuchung eine «sofortige Umplazierung [sic] zur Mutter». Das Vorhaben wurde in der Folge aber durch den «Widerstand des Knaben und der Pflegefamilie» verhindert, worauf die Assistenzärztin der Zweigstelle Winterthur dem Vormund eine stationäre Begutachtung in der Brüschhalde nahelegte. ${ }^{59}$ Roland konnte jedoch erst ein halbes Jahr später eingewiesen werden, weil sich die Pflegeeltern gegen einen stationären Aufenthalt «stemmten». Es brauchte nicht nur seitens des Vormunds Überzeugungskraft, er hatte darüber hinaus neben einem Mitglied der Vormundschaftsbehörde eigens einen Stadtrat für ein Gespräch mit den Pflegeltern aufgeboten, um deren Einwilligung zu erhalten. In dieser Zeit durfte die Mutter keinen Kontakt zu Roland haben. ${ }^{60}$

Die im Gutachterauftrag formulierte Fragestellung lässt den hohen Stellenwert des Kindeswohls für den Vormund erkennen. Er bat die Leitung der

58 Vgl. Galle 2016, 624.

59 StAZH, Z 98, Dossier Nr. 3483, Bericht Assistenzärztin der Zweigstelle Winterthur an Amtsvormund, 8.6.1971.

60 StAZH, Z 98, Dossier Nr. 3483, Gutachten vom 07.06.1972, 3, und Krankengeschichte, 3r, Eintrag Aus den Akten der V[ormundschaftsbehörde] Winterthur, 16.2.72. 
Beobachtungsstation nicht nur um eine Beurteilung der «Persönlichkeit» seines Mündels und fragte danach, wie sich eine Umplatzierung zur Mutter auf dessen «künftige Entwicklung» auswirken werde. Er wollte zudem wissen, wie und wann sich diese «am schonendsten» für den Knaben vornehmen liesse und wie die künftigen Kontakte zur Pflegefamilie geregelt werden könnten. Falls eine Umplatzierung vorläufig nicht zu verantworten wäre, wünschte sich der Vormund Angaben dazu, wie die Kontakte zur Mutter geregelt werden sollten. Die Pflege und Erziehung des Buben in der Pflegefamilie war seiner Einschätzung nach in Ordnung. Das Anliegen der Mutter, das Kind zu sich zu nehmen, erschien ihm vielmehr aufgrund ihrer veränderten Lebensumstände berechtigt. Für das behördliche Vorgehen sollte der ärztliche Bericht wie im Fall von Anna ebenfalls handlungsleitend sein. Gleichzeitig bedeutete die stationäre Begutachtung auch eine entlastende Massnahme in einer konfliktiven Situation.

Obwohl sich weder die Begutachtungspraxis in der Brüschhalde noch das Krankheitsbild der «neurotischen Reaktionen» in den 15 Jahren, die zwischen den behördlichen Aufträgen liegen, grundlegend verändert hatten, zeigen sich deutliche Unterschiede in den Gutachten, wie aus den folgenden Ausführungen hervorgeht. Sie waren vornehmlich aussermedizinischen Faktoren geschuldet. Der ärztliche Blick auf das Kind war bei der Begutachtung von Roland massgeblich geprägt durch die aufgrund der Heirat veränderten rechtlichen, sozialen und ökonomischen Verhältnisse der Kindsmutter. Die Erziehungsfähigkeit der Mutter blieb aus psychiatrischer Sicht allerdings, wie noch zu zeigen sein wird, ungewiss und eine potentielle Gefährdung für das Kind. Bei Anna wurde der Einbezug der Mutter hingegen zu keinem Zeitpunkt auch nur erwogen.

\section{7 - Abnorme Reaktionen als Ausdruck eines «schwierigen Kindes»}

Anna wurde am 29. Januar 1957, bereits zwei Wochen nachdem der Amtsvormund die Leitung der Brüschhalde um Aufnahme seines Mündels gebeten hatte, von ihrer Pflegmutter in die Kinderbeobachtungsstation gebracht. Rund vier Monate später lag der ärztliche Bericht vor, verfasst von einer Assistenzärztin und mit Unterschrift genehmigt von Oberarzt Corboz. Dass Ärztinnen und Ärzte in Ausbildung mit der psychiatrischen Begutachtung der Kinder betraut wurden, stellte in der klinischen Psychiatrie die Regel dar, widersprach indes der Lehrmeinung, gemäss der die «analytische Ausforschung» der Patientinnen und Patienten umfassendes Wissen erfordert 
und grosser Erfahrung bedarf. ${ }^{61}$ Wie in der Erwachsenenpsychiatrie erlernten die Berufsanfängerinnen und -anfänger in der Brüschhalde «ihr Handwerk» erst im klinischen Alltag. ${ }^{62}$ Die «häufig wechselnden und wenig erfahrenen Assistenzärzte» wurden bereits in der Vorgängerinstitution als Problem wahrgenommen. ${ }^{63}$ Die Assistenzärztin hatte zuvor immerhin während zweier Monate in der kinderpsychiatrischen Poliklinik einschlägige Erfahrungen sammeln können. ${ }^{64}$

Herleitung und Plausibilisierung einer lehrbuchmässigen Diagnose

Das dreiseitige Gutachten über Anna beginnt mit dem Einweisungsgrund und der Diagnose als Ergebnis der klinischen Begutachtung. Es folgen die Schlussfolgerungen aus der Anamnese und der Exploration, das heisst den Erhebungen, Untersuchungen und Beobachtungen im Heim, zu denen verschiedene Testverfahren gehörten. Das Gutachten schliesst mit einer ärztlichen Prognose zur erwarteten Entwicklung des Kindes und, wie vom Amtsvormund gewünscht, einer Empfehlung zur Platzierung. Das Gutachten enthält damit alle üblichen Bestandteile. Dass die Diagnose am Beginn steht, impliziert deren narrative Herleitung und Plausibilisierung.

Bei den «zunehmende[n] Schwierigkeiten», die Anna laut ihrem Vormund in der Pflegefamilie bereitet hatte, handelte es sich aus Sicht der Assistenzärztin um «neurotische» Reaktionen. Als erstes fällt auf, dass die Erziehungsschwierigkeiten des Kindes Ausgangspunkt blieben, obwohl die Assistenzärztin die Pflegefamilie kritisch beurteilte. Die Pflegemutter sei eine «nervöse, überempfindliche Frau», «welche erzieherisch ungeschickt und überfordernd dem Kinde gegenüberstand und ihm keine richtige Geborgenheit geben konnte». Es sei nachvollziehbar, dass sich das Mädchen «immer mehr vereinsamt, bedroht» fühlte. ${ }^{65}$ Dass sich die ärztliche Beschreibung des kindlichen Verhaltens und Charakters gleichwohl auf die Aussagen der Pflegemutter abstützte, verdeutlicht den eminenten Stellenwert der «Vorgeschichte» in der psychopathologischen Begutachtung. Gemäss dem Kinderpsychiater Erich Benjamin war ein «wirkliches Verständnis des schwierigen Kindes [...] ohne genaue Kenntnis seiner Vorgeschichte nicht möglich».6 ${ }^{66}$ Die Anamnese nahm auch bei der Begutachtung in der Brüschhalde einen zentralen Stellenwert ein. Über Anna hielt die Assis-

61 Tramer 1949, 130.

62 Bühler et al. 2019, 339; Jenzer et al. 2017, 120, 129.

63 Zürrer-Simmen 1994, 70.

64 KPD, Jahresbericht 1956, 9, und Jahresbericht 1957, 2 , 9.

65 StAZH, Z 98, Dossier Nr. 2422, Gutachten vom 20.5.1957, 1.

66 Benjamin 1938, 159. 
tenzärztin in ihrem Gutachten fest, das Mädchen sei insbesondere auf die Pflegetochter und ihren Stiefbruder «eifersüchtig» gewesen. Sie habe angefangen zu «trotzen», mit Tages- und Bettnässen «auf noch sehr kleinkindlicher Stufe Aggressionen» geäussert und «Nägelbeissen» aufgewiesen. Durch seine «Geltungssucht» habe sich das Mädchen unbeliebt gemacht, es habe «Mühe mit dem Kontaktaufbau» gehabt und sich «eher scheu und verschlossen» verhalten.

Die Assistenzärztin vermerkte damit exakt diejenigen Verhaltensweisen und Eigenschaften im Gutachten, die «neurotische» Reaktionen lehrbuchmässig auszeichneten. So bestimmten laut den Ausführungen zur kindlichen Neurose im damaligen Standardlehrbuch für Psychopathologie «Trotz», «Abwendung» und «Regression» die Entwicklung des «schwierigen Kindes». Diese «drei Reaktionen» bildeten gemäss Benjamin die «Grundlage einer Diagnostik des Wesens schwieriger Kinder». ${ }^{67}$

Aus pädiatrischer Sicht handelte es sich bei «neurotischen Reaktionen» um Funktionsanomalien des Nervensystems, die vorwiegend auf erblicher Belastung beruhten. Die Entstehung «neurotischer» Erscheinungen, Mechanismen bzw. Reaktionen - je nach fachärztlicher Terminologie - war indes, das betont auch Benjamin, wesentlich abhängig von Umwelteinflüssen. ${ }^{68}$ Demnach konnten «neurotische» Reaktionen durch die Einwirkung des Milieus beziehungsweise des Umfeldes ausgelöst, verstärkt oder gemindert werden. Hierzu bleibt das Gutachten über Anna jedoch auffällig vage. In welcher Hinsicht sich die erwähnten «viel[en] Wechsel», die das Kind anscheinend «in den entscheidenden Jahren» erlebt hatte, negativ auswirkten, führte die Assistenzärztin nicht aus. Auch worin das angebliche «Verwöhnen» durch die Grossmutter bestand, bei der es vor der Fremdplatzierung aufwuchs, und welche Folgen dieses für das Kind hatte, wird nicht dargelegt.

Warum Anna zwischen den «verschiedenen Mutterfiguren», womit vermutlich ihre Mutter und Grossmutter gemeint waren, hin- und hergerissen war und was dies für das Kind bedeutete, bleibt allenfalls zu erahnen. Auch hier schafft ein Blick ins Lehrbuch Klärung: So galten die «verwöhnende», die «sprunghafte» und die «überfordernde» Erziehung wie auch die vaterund mutterlose Erziehung als «ungünstige Umwelteinflüsse». Für eine «normale» Entwicklung des Kindes erachtete die Ärzteschaft die «Harmonie des Familienlebens» als «unentbehrlich». ${ }^{69}$

67 Benjamin 1938, 187. Moritz Tramer kritisierte die Gleichsetzung der Reaktionstypen mit Erziehungsschwierigkeiten, erachtete die Klassifikation aber dennoch als gute Orientierung. Vgl. Tramer 1949, 393.

68 Benjamin 1938, 154.

69 Benjamin 1938, 168-175; Lutz 1961, 48-58. 
Neben den «hereditären [d.h. erblich bedingten] Verhältnisse[n]» fokussierte die psychiatrische Anamnese auf den «individuellen Lebens- und Entwicklungsablauf» des Kindes..$^{70}$ Als Massstab diente die «normale», in Altersstufen eingeteilte Entwicklung des Kindes und der idealtypische Lebensverlauf in einer bürgerlichen Kleinfamilie. Was der Arzt oder die Ärztin beobachtete, waren die «abnorme[n] Formen» insbesondere der «kritischen Entwicklungsphasen». ${ }^{71}$ Kinder mit normabweichenden, in damaligen Worten «abnormen» Verhaltensweisen und Charaktereigenschaften wurden als «schwierige» und «anormale» Kinder bezeichnet. ${ }^{72}$

Auffallend ist, dass sich die genannten Zuschreibungen und angenommenen Folgen mangelnder elterlicher Präsenz auch in den Testresultaten wiederfinden, aus denen die Assistenzärztin eine ausgeprägte Entwicklungshemmung folgerte. So heisst es im Gutachten: «In verschiedenen Tests tritt eine gute Begabung mit einem erheblichen Infantilismus zu Vorschein.» ${ }^{73}$ Die Assistenzärztin hatte mit Anna, wie es in der Brüschhalde üblich war, die damals in der Kinderpsychiatrie gebräuchlichen Tests durchgeführt, um Aufschluss über die Intelligenz, die Leistungsfähigkeit und die Persönlichkeitsentwicklung des Kindes zu erhalten. ${ }^{74}$ Während die Auswertung des Intelligenztests eine leicht über der Norm liegende, «gut durchschnittliche Intelligenz» ergab, attestierte die Assistenzärztin Anna anhand des Rorschach- und des Sceno-Tests - allerdings erst gegen Ende des Beobachtungsaufenthalts aufgrund einer zweiten Testserie - eine Entwicklungshemmung, wie sie nach damaliger Lehrmeinung für «neurotische» Reaktionen kennzeichnend war. ${ }^{75}$

Die beiden angewandten assoziativen Testverfahren hingen weitgehend von subjektiven Einschätzungen ab. Sowohl der Rorschachtest, bei dem durch die Deutung von Tintenklecks-Faltbildern Angaben zur Persönlichkeit gesucht werden, wie auch der projektive Sceno-Test, bei dem aus dem kindlichen Spiel mit vorgegebenem Material auf dessen innere Konflikte geschlossen wird, waren und sind aufgrund ihrer mangelnden Reliabilität umstritten, fanden aber im Untersuchungszeitraum standardmässig Anwendung. ${ }^{76}$ Aus den stichwortartigen, in der Krankenakte überlieferten Notizen zu den Tests sind die Schlussfolgerungen nicht ohne Weiteres nachzuvollziehen. Es bleibt somit offen, warum der Assistenzärztin die Entwicklungshem-

70 Tramer 1949, 123.

71 Tramer 1949, 19.

72 Benjamin 1938, 159.

73 StAZH, Z 98, Dossier Nr. 2422, Gutachten vom 20.5.1957, 2. Infantilismus bezeichnete in der damaligen Psychiatrie eine Entwicklungshemmung. Vgl. Tramer 1949, 376.

74 Vgl. zur Übersicht Hiltmann 1960 und Herzka 1978.

75 Vgl. auch Lutz 1961, 307.

76 Germann 2019; Hiltmann 1960, 220. 
mung besonders ausgeprägt erschien. Ihre Deutung steht aber im Gegensatz zu den in der prognostischen Bewertung erwähnten Eigenschaften des Mädchens, die als positiv hervorgehoben und an erster Stelle erwähnt werden, nämlich das «kräftige Reaktionsvermögen» und die «aktive Auseinandersetzungsmöglichkeit». ${ }^{77}$

Strukturbildend für das Narrativ war die «fundamentale Tatsache, dass die Grundlagen der Schwererziehbarkeit immer in der frühen Kindheit zu suchen» und Neurosen zwar stets durch das Milieu ausgelöst wurden, nach damaliger Auffassung aber mehrheitlich durch ererbte und angeborene Veranlagungen bedingt oder zumindest begünstigt waren. Dies obwohl weder die Annahme der frühkindlichen Prägung noch der Erbgang wissenschaftlich «völlig klargestellt», das heisst erwiesen waren. ${ }^{78}$ Sie erhielten kraft ärztlicher Autorität ihre Gültigkeit und stellten eine Tatsache dar, die es nur noch zu belegen galt. Genau diese Funktion erfüllte das Gutachten über Anna. ${ }^{79}$

Familienverhältnisse als Krankheitsursache

«[N]egative Elemente» der kinderpsychiatrischen Prognose stellten «der frühe Beginn und die lange Dauer der Schädigung, sowie die Unabänderlichkeit der Verhältnisse» dar. Liest man Annas Krankengeschichte, die einen Auszug aus den Vormundschaftsakten enthält, so fällt auf, dass sich die «Schwierigkeiten»- anders als es der Amtsvormund darstellte - erst in der Pflegefamilie in Bülach zeigten. Als Anna noch bei ihrer Grossmutter lebte, wurde sie von ihrem Vormund «als gesund und munter taxiert». Nachdem es $\mathrm{zu}$ «Reibereien» zwischen der Mutter und Grossmutter des Kindes gekommen war, zeigte sich letztere einverstanden, das Kind in eine Pflegefamilie zu geben. Das als «fröhlich», «lebhaft» und «intelligent» beschriebene Kind bereitete in der ersten Pflegefamilie «nie besondere Schwierigkeiten». Die Umplatzierung erfolgte, weil die in Ausbildung befindliche Tochter des Hauses Ruhe zum Lernen brauchte. ${ }^{80}$ Dennoch ging die Assistenzärztin von einer frühen Schädigung des Kindes aus. Das Gespräch mit der Pflegemutter aus Bülach hatte stattgefunden, bevor die Assistenzärztin die Akten eingesehen

77 StAZH, Z 98, Dossier Nr. 2422, Gutachten vom 20.5.1957, 3.

78 Benjamin 1938, 164, 188. Auch Lutz weist darauf hin, dass die Krankheitsursachen «vielfältig und noch nicht bis in Letzte abgeklärt» seien. Vgl. Lutz 1961, 301, 308.

79 Zur Herstellung und Zirkulation von ärztlichem Wissen und zum autoritativ-normativen Anspruch der Wissenschaft vgl. Meier 2006, Imboden/Ritter 2008 und Tanner 2007a.

80 StAZH, Z 98, Dossier Nr. 2422, Krankengeschichte, 8, Eintrag vom 7.2.1957. 
hatte. ${ }^{81}$ Die Diagnose stand für sie offensichtlich bereits zu Beginn der Begutachtung fest und wurde nicht mehr präzisiert.

Stringenz konnte die Assistenzärztin nur erzeugen, indem sie die in der Anamnese erhobenen, heterogenen Angaben selektionierte. So blieben auch weitere positive Aussagen unerwähnt, wie zum Beispiel von Annas Lehrerin. Während die Pflegemutter das Kind «als wahres Teufelchen und unausstehlichen Tyrannen schilderte», machte Anna auf die Lehrerin einen «frischen, fröhlichen Eindruck, ihr hübsches Äusseres erweckte unwillkürlich Sympathie».82

Dass das Verhalten von Anna als pathologisch taxiert wurde, ist massgeblich dem kinderpsychiatrischen Begutachtungsverfahren und den Annahmen über die familiären Verhältnisse geschuldet. Anna wurde nie direkt zu ihrer Situation befragt. Das Gutachten stellte für die kindliche Perspektive vollumfänglich auf Testresultate sowie Berichte der heimeigenen Lehrpersonen und der Erzieherin ab. Die Befunde der verschiedenen Testverfahren lassen sich indes nicht schlüssig von den Resultaten ableiten. Sie bestätigten vielmehr weitgehend die Vorannahmen. Die Aussagen des Kindes wurden zu blossen Symptomen «neurotischer» Reaktionstypen. «Trotzen» und «Bettnässen» konnten in der Brüschhalde zwar nicht beobachtet werden. Das Mädchen erwies sich aber angeblich auch dort als «überempfindlich, sehr geltungsbedürftig».83 Anna wurde in der kinderpsychiatrischen Beobachtungsstation schliesslich nur noch mit ihrer Diagnose identifiziert. ${ }^{84}$

«Neurotische» Reaktionen waren gemäss Moritz Tramer pathologische Reaktionen auf «Konflikte und Insulte». ${ }^{85}$ Auch gemäss Jakob Lutz gab es keine «neurotischen Reaktionen» ohne «entsprechende traumatisierende Umwelterlebnisse». ${ }^{86}$ Obwohl die Assistenzärztin die fehlerhafte Erziehung der Pflegemutter im Gutachten nicht als auslösendes Moment identifizierte, sondern die «neurotischen» Reaktionen auf eine frühkindliche, d.h. in den ersten drei Lebensjahren erfolgte Schädigung zurückführte, hielt sie fest, dass diese bei einer Rückkehr in die Pflegefamilie «sicher in kürzester Zeit» wieder auftreten würden. ${ }^{87}$

81 StAZH, Z 98, Dossier Nr. 2422, Krankengeschichte, 8, Eintrag vom 29.1.1957.

82 Zur Tendenz der Pathologisierung von Kindern und Jugendlichen anstelle der Problematisierung von Fremdplatzierungssituationen vgl. auch Janett 2018, 263.

83 StAZH, Z 98, Dossier Nr. 2422, Gutachten vom 20.5.1957, 2.

84 Vgl. Goffman 1973, 51.

85 Tramer 1949, 394.

86 Lutz 1961, 308; Lutz 1972, 322.

87 StAZH, Z 98, Dossier Nr. 2422, Gutachten vom 20.5.1957, 3. 
Die «Prognose dieser neurotischen Reaktionen» bleibt im Gutachten offen. Die als positiv bezeichneten Faktoren vermochten die negativen nicht aufzuwiegen. Die Pathologisierung des «illegitimen Mädchens» war wie oben beschrieben eine direkte Folge seiner aus Sicht der Assistenzärztin unabänderlichen rechtlichen, sozialen und ökonomischen Situation. «Wie weit bei der pathologischen Persönlichkeit der Mutter und des Vaters» die erwähnten Symptome des Mädchens «teilweise konstitutionell bedingt sind», war gemäss der Assistenzärztin derzeit nicht «sicher zu entscheiden». ${ }^{88}$ Es erschien damit durchaus möglich, dass sich das «schwierige» Kind, wie in der damaligen Psychiatrie häufig beobachtet, zu einer «abnormen Persönlichkeit» entwickelte. ${ }^{89}$

Anna sollte nach Ansicht der Assistenzärztin «nun zuerst die Möglichkeit bekommen, eine tiefere Beziehung zu einer Muttererscheinung aufzubauen». Erst auf Grund dieser Beziehung werde es möglich sein, «erzieherische Forderungen» an das Mädchen zu stellen. ${ }^{90}$ Das Ziel war nicht nur eine medizinische Besserung oder Heilung, die bei «kindlichen Neurosen schon durch einfache Milieuveränderung oder durch einfachste ärztliche Massnahmen» möglich erschien, ${ }^{91}$ sondern auch, die gesellschaftliche Anpassung und Nützlichkeit des Kindes herbeizuführen. ${ }^{92}$ Die Bedingungen dafür sah die Assistenzärztin «entweder in einer sehr qualifizierten, heilpädagogisch begabten Pflegefamilie oder einem kleineren, familiär geführten Heim» als erfüllt. Zudem sollte «die Möglichkeit einer ergänzenden Spieltherapie» ins Auge gefasst werden..$^{93}$ Die «tiefere Beziehung» des Kindes zur Grossmutter aufrecht zu erhalten, zog die Assistenzärztin nicht in Betracht, obwohl die Grossmutter für Anna «immer nett gesorgt» und ihr Enkelkind «regelmässig» besucht hatte, wie sie in der Krankenakte vermerkte. ${ }^{94}$ Aus psychiatrischer Sicht waren Grosseltern aus verschiedenen Gründen eher ungeeignete Erziehungspersonen. ${ }^{95}$ Bei Anna hätte eine Rückkehr zur Grossmutter der konstatierten «frühkindlichen Schädigung» widersprochen.

Die Mutter des Mädchens galt als «unstet», «arbeitsscheu» und «unzuverlässig». Sie war 1954 wegen «liederlichen Lebenswandels» für ein Jahr in eine Arbeitsanstalt eingewiesen worden, nachdem bei ihr aufgrund einer Be-

88 StAZH, Z 98, Dossier Nr. 2422, Gutachten vom 20.5.1957, 3.

89 Tramer 1949, 394.

90 StAZH, Z 98, Dossier Nr. 2422, Gutachten vom 20.5.1957, 3.

91 Benjamin 1938, 165.

92 Zur «sozialen Heilung» als Ziel psychiatrischer Therapien vgl. Meier 2004.

93 StAZH, Z 98, Dossier Nr. 2422, Gutachten vom 20.5.1957, 3.

94 StAZH, Z 98, Dossier Nr. 2422, Krankengeschichte, 5.

95 Lutz 1961, 53. 
gutachtung in der psychiatrischen Universitätsklinik Waldau in Bern eine «Psychopathie» diagnostiziert worden war. ${ }^{96}$ Wie neuere Untersuchungen zeigen, wurden Kinder von Eltern, die ihrerseits schon von fürsorgerischen Zwangsmassnahmen betroffen waren, überproportional häufig begutachtet und fremdplatziert und waren wie Anna vor physischer und psychischer Gewalt unzureichend oder gar nicht geschützt. ${ }^{97}$ Anna wurde von ihrer Pflegemutter nicht nur verunglimpft und erlebte die Situation in der Pflegefamilie wie im Gutachten beschrieben als bedrohlich, sondern wurde auch geschlagen. Laut der Pflegemutter gab es «sehr selten Tätsch, keine richtigen Schläge». Die Nachbarn vermuteten allerdings, es werde «sicher zu oft geschlagen $»{ }^{98}$ Weder physische noch psychische Gewalt wurde im Gutachten problematisiert. In der medizinisch-psychiatrischen Begutachtung von Kindern und Jugendlichen wie auch bei den Behörden wurde Gewalt erst viel später ein relevantes Thema. ${ }^{99}$

Die Pathologisierung des Kindes entlastete den Vormund vielmehr von der möglichen Kritik in einem Rechtsmittelverfahren, das Mädchen am falschen Ort untergebracht zu haben. ${ }^{100}$ Er war an der ärztlichen Besprechung, an der die Assistenzärztin dem Oberarzt die Diagnose und die Therapie erörterte, anwesend. ${ }^{101}$ Das anschliessend verfasste psychiatrische Gutachten konnte vor diesem Hintergrund gar nicht, wie von Gesetzes wegen vorgesehen, ein mögliches Korrektiv darstellen. ${ }^{102} \mathrm{Im}$ Gegenteil entlastete es den Vormund auch künftig, die von ihm angeordneten Massnahmen kritisch zu reflektieren. Die Ursachen der Erziehungsschwierigkeiten waren aufgrund der Diagnose beim Kind zu suchen.

\section{2 - Abnorme Reaktionen als Ausdruck einer «schwierigen Situation»}

Das Gutachten, das am 7. Juni 1972, am letzten Tag von Rolands viereinhalbmonatigem Aufenthalt in der Brüschhalde seinem Vormund verschickt wurde, umfasst 14 Seiten und fällt somit deutlich umfangreicher aus als der bloss dreiseitige Bericht über Anna. Die Gutachten in der Brüschhalde haben - wie auch in den psychiatrischen Kliniken - eine erhebliche Varianz im

96 StAZH, Z 98, Dossier Nr. 2422, Krankengeschichte, 5.

$97 \mathrm{Vgl}$. Ammann/Schwendener 2019, 21-59.

98 StAZH, Z 98, Dossier Nr. 2422, Krankengeschichte, 7, Eintrag vom 29.1.1957; 8, Eintrag vom 7.2.1957.

99 Businger/Ramsauer 2019, 40-48.

100 Der Vormund bzw. die zuständige Behörde musste vor der Bewilligung zur Aufnahme eines Kindes die Eignung der Pflegeeltern prüfen. Vgl. Egger 1943, 112.

101 StAZH, Z 98, Dossier Nr. 2422, Krankengeschichte, 8, Eintrag vom 14.5.1957.

102 Vgl. Egger 1943, 104. 
Umfang. Dieser nahm aber im untersuchten Zeitraum tendenziell zu. ${ }^{103}$ Der eher überdurchschnittliche Umfang des Gutachtens über Roland ist zum einen dem Umstand geschuldet, dass dessen Vormund präzise Fragen stellte, die auf der ersten Seite referiert werden. Darauf folgt eine Zusammenstellung der zahlreichen Unterlagen, auf die sich das Gutachten abstützt. Zum anderen ist der Aufbau des Gutachtens ein anderer als bei Anna. Das Gutachten beginnt ebenfalls mit dem Anlass, der zur Begutachtung führte. Anschliessend werden aber die Familienanamnese und die persönliche Anamnese von Roland auf zwei Seiten dargelegt und es folgen mehr als vier Seiten eingehende Ausführungen zu den eigenen Untersuchungen und Befunden. Es werden ausserdem die Ergebnisse der einzelnen Testverfahren festgehalten, von denen nicht weniger als 14 verschiedene durchgeführt wurden. Daran schliesst das eigentliche Gutachten an, das die vorangehenden Ausführungen zusammenfasst und die vom Amtsvormund gestellten Fragen beantwortet. Die Diagnose findet sich, ohne als solche explizit ausgewiesen zu sein, erst in den Antworten. Der begutachtende Assistenzarzt hielt fest, dass im Zusammenhang mit der versuchten Umplatzierung von Roland zu seiner Mutter «neurotische und psychosomatische Reaktionen» aufgetreten seien. Das Gutachten hatte wie bei Anna 15 Jahre zuvor Jules Robert Corboz autorisiert, der nun als Direktor des kantonalen Kinderpsychiatrischen Diensts der stationären Beobachtungsstation vorstand.

\section{Die Persönlichkeit der Mutter im Fokus}

Die Anamnese basierte im Unterschied zum Gutachten über Anna nicht nur auf einem Gespräch mit den Pflegeeltern und der Einsicht in die Akten der Vormundschaftsbehörde, sondern auch auf den mündlichen Auskünften der Mutter und ihres Ehemannes sowie auf mündlichen und schriftlichen Angaben der stationseigenen Fürsorgerin. Einbezogen war zudem der kinderpsychiatrische Bericht der Zweigstelle Winterthur, wo Roland zuvor ambulant untersucht worden war. Wie aus der Krankengeschichte der Brüschhalde hervorgeht, fand nicht nur ein Anamnesegespräch mit der Mutter und dem Stiefvater statt. Die Mutter durfte ihren Sohn überdies zweimal in der Brüschhalde besuchen und mit ihm einen Ausflug machen, woraus sich weitere Gespräche mit dem Assistenzarzt ergaben. ${ }^{104}$

103 Eigene Erhebung (Anm. 14). Vgl. auch Bühler et al. 2019, 327.

104 StAZH, Z 98, Dossier Nr. 3483, Krankengeschichte, 2, Eintrag Besuch von Km und Stv, 15.03.1972; 2r, Eintrag 2. Besuch von Km und Stv, 20.04.1972; 6r, Eintrag Provisorische Zusammenfassung meiner Eindrücke vom Pat. in den letzten Wochen, 02.05.1972; 7r, Eintrag Besuch von Km und Stv, 25.05.1972. 
Wie Annas Mutter wuchs auch die Mutter von Roland bei Eltern mit einer «lange Zeit zerrütteten, später geschiedenen Ehe» auf. Erwähnt ist sodann, dass sie ein Jahr in einem Mädchenheim untergebracht war und an verschiedenen Stellen in Haushalten und Fabriken gearbeitet hatte. Was der Mutter von Anna noch als unsteter Lebenswandel ausgelegt worden war, wird ebenso nüchtern beschrieben wie die von den Pflegeeltern vorgebrachten und vom Vormund bestätigten früheren «verschiedene[n] kurzdauernde[n] Bekanntschaften». ${ }^{105}$ Dies, obwohl die Pflegeeltern die Mutter wiederholt als «sexuell haltlos» und «labil» diffamierten und ihr vorwarfen, sie habe «keine mütterlichen Gefühle». ${ }^{106}$ Entscheidend für den Gutachter war offenbar, dass dem Vormund in «den letzten Jahren keine ungünstigen Angaben über die K[inds]m[utter] von Drittpersonen zu Ohren gekommen» waren. ${ }^{107}$

Die Eindrücke über die Mutter und den Stiefvater, die der Assistenzarzt in der Krankengeschichte festhielt, waren im Vergleich zum Gutachten indes noch deutlich stärker wertend. So war der Stiefvater in seinen Augen «eher weich und nicht allzu durchsetzungsfähig und belastbar, nicht überaus intelligent». Er machte dennoch einen guten Eindruck, weil er «anscheinend offen, gutmeinend, um Roland echt bemüht ist, ihn wirklich gern hat». Auch die Mutter von Roland erschien dem Assistenzarzt «nicht sehr differenziert und ziemlich unselbständig». Dass sie trotzdem «verständig über Probleme», die ihren Sohn betrafen, mit ihm sprach, veranlasste ihn zu der Frage, ob dies «angelernt» sei. ${ }^{108}$ Der Assistenzarzt war offensichtlich bemüht, «echte» von vorgespielten Gefühlen und instinktives von gelerntem Verhalten zu unterscheiden. Im Gutachten heisst es schliesslich, die Kindsmutter habe den Eindruck «einer etwas unsicheren, noch nicht sehr gereiften Frau» hinterlassen. Ihr Ehemann sei ein «tüchtiger Maschinenschlosser», der als städtischer Angestellter arbeite. Die Beiden lebten in einer 4-Zimmer-Wohnung und die finanziellen Mittel seien ausreichend. ${ }^{109}$

Für die Erfassung der Persönlichkeit der Eltern verfügte die Kinderpsychiatrie über keine disziplinären Kriterien. Entscheidend war vielmehr der Eindruck, den diese beim untersuchenden Assistenzarzt hinterliessen. ${ }^{110}$ Auch die detaillierte physische Beschreibung in der Krankengeschichte

105 StAZH, Z 98, Dossier Nr. 3483, Gutachten vom 07.06.1972, 3.

106 StAZH, Z 98, Dossier Nr. 3483, Bericht des Pflegevaters über die Mutter von Roland, 8 Seiten, und Zusammenfassung der gemeinsamen Besprechung, 04.05.1971.

107 StAZH, Z 98, Dossier Nr. 3483, Gutachten vom 07.06.1972, 3.

108 StAZH, Z 98, Dossier Nr. 3483, Krankengeschichte, 2, Eintrag Besuch von Km und Stv, 15.03.1972; 1r, Eintrag Gespräch mit der Km in der Poliklinik, 02.02.1972.

109 StAZH, Z 98, Dossier Nr. 3483, Gutachten vom 07.06.1972, 3.

110 Dies bestätigte der Kinderpsychiater Heinz Stefan Herzka, der 1964 als Assistenzarzt in der Brüschhalde tätig war, in einem persönlichen Gespräch am 22. Januar 2020 in Zürich. Vgl. bereits Tramer 1949, 123. 
zeigt den Blick eines Akademikers auf ein Paar mit niedrigerem sozialen Status, das aber seit der Heirat einen ordnungsgemässen zivilrechtlichen Status hatte und bei dem mindestens der Mann die ihm zugedachte Rolle des Ernährers und Familienoberhaupts erfüllte. Ob Rolands Mutter den gesellschaftlichen Erwartungen einer Frau, Ehefrau und Mutter nachkam, versuchte die Fürsorgerin bei ihrem Hausbesuch herauszufinden. Dass diese von der Haushaltsführung auf die Persönlichkeit und damit Eignung als Mutter schloss, entsprach durchaus der damaligen fürsorgerischen Praxis. ${ }^{111}$

Auffallend ist, dass der Bericht der Fürsorgerin ebenfalls kritischer ausfällt als die Darstellung im Gutachten. Es zeigt sich zudem, dass die Schilderungen der Pflegeeltern und die Einschätzungen des Assistenzarztes die Überlegungen der Fürsorgerin beeinflussten, was in der Übernahme der psychiatrisch-psychologischen Terminologie zum Ausdruck kommt. ${ }^{112}$ Deutlich wird aber auch, dass der Bericht der Fürsorgerin nicht dieselbe Stringenz erforderte wie das kinderpsychiatrische Gutachten, sondern Abwägungen zuliess und Platz für persönliche Ansichten bot, die auch als solche erkennbar sind. Obwohl die Pflegeeltern «eher Zwangscharaktere» seien und «sehr w[ahrscheinlich] eine überstarke Bindung» an die Pflegemutter bestehe, glaubte die Fürsorgerin, dass Roland «gemütsmässig» bei ihnen mehr bekomme als von seiner «eher dürftig 'angereichert[en]'» Mutter. Einen «Wechsel in der ödipalen Phase» hielt die Fürsorgerin für ungünstig. «Triftige Gründe», um der Mutter das Kind zu verweigern, machten sich während des Besuches aber «nicht bemerkbar». Die Fürsorgerin gab jedoch zu bedenken, dass die Prognose für Roland in Anbetracht der «Anamnese der K[inds]$\mathrm{m}$ [utter]» nicht günstig sei, insbesondere wenn die Aussagen des Pflegevaters der «Realität» entsprechen sollten. Gleichzeitig war für sie denkbar, dass eine Platzierung des Kindes bei der Mutter «ev. im Gegenteil eine günstige Wirkung auf den schon gepflanzten 'Zwangscharakter'» haben könnte. ${ }^{113}$

Die Pflegefamilie führte laut dem ärztlichen Gutachten «ein geordnetes, harmonisches Familienleben». Das Verhältnis zur Kindsmutter wird als angespannt beschrieben. Sie sei in die Rolle der «unbotmässigen Tochter» geraten. Gegenüber dem Knaben hätten sich die vorzeitig gealterten Pflegeltern wie «überaus bemühte» Grosseltern verhalten. Erzieherisch habe Roland keine Schwierigkeiten geboten. ${ }^{114}$ Der in der Krankengeschichte festgehaltene und sich im Laufe der Beobachtung für den Assistenzarzt bestätigende Eindruck,

111 Businger/Ramsauer 2019, 30-34; Ramsauer 2000, 126-133.

112 Die Fürsorgerin war an der «Gemeinsamen [Besprechung]» beteiligt und hatte höchstwahrscheinlich auch Einsicht in die Krankengeschichte. Sie benutzte teilweise identische Formulierungen wie der fallführende Assistenzarzt.

113 StAZH, Z 98, Dossier Nr. 3483, Hausbesuch bei K[inds]e[eltern], 16.02.1972, 1-3.

114 StAZH, Z 98, Dossier Nr. 3483, Gutachten vom 07.06.1972, 3-5. 
auf den sich auch die Fürsorgerin bezieht, die Pflegeltern «seien recht zwangsneurotische Charaktere [mit einengender Erziehungshaltung]», ${ }^{115}$ fand im Anamneseteil des Gutachtens keinen Niederschlag. Erst in der Darlegung der eigenen Befunde und bei der Beantwortung der behördlichen Fragen am Schluss des Gutachtens wird eine kritischere Wahrnehmung ersichtlich. So wird der Grund für den geschilderten Rückstand in der Persönlichkeitsentwicklung des Knaben vor allem in der «überbehütenden Haltung» der Pflegemutter verortet. ${ }^{116}$ Es war also nicht allein das in allen Lehrbüchern unter «Störfaktoren» stets an erster Stelle erwähnte «geordnete Familienleben», das als Grundvoraussetzung für eine «normale» Entwicklung des Kindes galt. ${ }^{117}$ Vielmehr war eine «genaue Krankheitserkenntnis und eine wirksame Behandlung [...] undenkbar ohne ganz spezielle Untersuchungen und Beeinflussungen des Milieus». ${ }^{118}$ Im Zentrum des kinderpsychiatrischen Fokus stand dabei die Persönlichkeit der Mutter bzw. der Mutterfigur und ihre Beziehung zum Kind. ${ }^{119}$

\section{Trotz «abnormer Reaktionen» keine Erziehungsschwierigkeiten}

Roland hatte sich laut dem Gutachten bald gut eingelebt in der Brüschhalde. Er bot im Unterschied zur Schilderung von Anna «erzieherisch keine Schwierigkeiten», erwies sich vielmehr als «anpassungsfähig, hilfsbereit und gut erzogen»- dies, obwohl sich auch bei Roland die typischen Symptome «neurotischer» Reaktionen zeigten: «Trotz», «Abwendung», «Regression» und «Aggression». ${ }^{120}$ So begann Roland in der Brüschhalde gemäss ärztlicher Ausführungen seine Trotzphase nachzuholen. Als «kleiner Wichtigtuer» und «Gernegross» habe er Gleichaltrigen in seiner phantasierten Stärke gedroht und auch handgreiflich werden können. In den Testergebnissen zeigte sich zudem eine «ausgeprägte Aggressivität» mit Ablehnung der Mutter und des Stiefvaters. Sein oft kleinkindlich-trotziges Verhalten in der Therapie war für den Assistenzarzt aber Ausdruck dafür, dass es gelungen sei, «die Fesseln der überbehütenden und einengenden Erziehung durch die Pfle[geeltern] zu sprengen»- und damit eine günstige Persönlichkeitsentwicklung zu fördern. Das Hin und Her zwischen Pflegefamilie und Mutter, heisst es weiter im Gutachten, habe bei Roland «schwere Existentialängste»

115 StAZH, Z 98, Dossier Nr. 3483, Krankengeschichte, 6r, Eintrag Provisorische Zusammenfassung meiner Eindrücke vom Pat. in den letzten Wochen, 02.05.1972.

116 StAZH, Z 98, Dossier Nr. 3483, Gutachten vom 07.06.1972, 12.

117 Benjamin 1938, 172-177; Lutz 1961, 48-58; Tramer 1949, 123-126.

118 Corboz 1969, 180; Lutz 1935, 4.

119 Vgl. Lutz 1961, 48.

120 Vgl. Lutz 1961, 307. 
geweckt, die er vor allem durch «Wunschphantasien» und «prahlerisches Sich-Aufspielen» abzuwehren versuchte. Seine «phobischen Verhaltensweisen» zeigten, dass sein Vertrauen in die Umwelt noch erschüttert sei. Wie bei Anna erkannte der Assistenzarzt sodann mangelndes Selbstwertgefühl. ${ }^{121}$

Im Vergleich zu 1957 führte der Assistenzarzt bei Roland 1972 eine deutlich grössere Zahl Tests durch. Dies entsprach einer generellen Entwicklung in der Kinderpsychiatrie jener Jahre. ${ }^{122}$ Das Testrepertoire umfasste neben einem Leistungs- und einem Intelligenztest einen Spezialtest zur Feststellung leichter frühkindlicher Hirnschädigungen sowie eine ganze Palette an sogenannten Entfaltungstests, als deren bekanntester der Rorschachtest zu nennen ist, der wie auch der Sceno-Test bereits bei Anna angewandt wurde. Diese Entfaltungstests, mit denen die altersgemässe Entwicklung geprüft wurde, bestätigten bei Roland ebenfalls die im Intelligenztest konstatierte leicht überdurchschnittliche Intelligenz. Der Assistenzarzt nahm bei der Auswertung der Tests ausserdem mehrfach explizit Bezug auf die Anamnese. ${ }^{123}$ Noch deutlicher als bei Anna wird ersichtlich, dass der Blick auf das kindliche Spiel und die während der Tests von Roland angefertigten Zeichnungen massgeblich auf den Erkenntnissen des Assistenzarztes über die Lebensgeschichte und die familiäre Situation des Kindes beruhte und die Testresultate erheblich beeinflusste. ${ }^{124}$ Die Tests sollten dem Assistenzarzt aber auch Aufschluss darüber geben, wie das Kind ein spezifisches Ereignis verarbeitete. In der Annahme, dass vor allem jüngere Kinder wie Roland noch nicht in der Lage seien, sich «auszusprechen», setzten Ärztinnen und Ärzte vor allem spielerische Tests ein. ${ }^{125}$ So mag der nach einem Ausflug von Roland mit seiner Mutter und dem Stiefvater durchgeführte Sceno-Test dazu beigetragen haben, dass der Assistenzarzt schliesslich die vom Vormund beabsichtigte Umplatzierung zur Mutter befürwortete. Gemäss den ärztlichen Aufzeichnungen gesellte sich zum Bild der bedrohlichen Mutter dasjenige «eines friedlichen, schönen Zusammenlebens mit K[inds]m[utter] und St[ief]v[ater]». ${ }^{126}$ Bemerkenswerterweise fand dieser Test jedoch im Gutachten kei-

121 StAZH, Z 98, Dossier Nr. 3483, Gutachten vom 07.06.1972, 6-10.

122 Während 1957 standardmässig vier bis sechs Tests durchgeführt wurden, waren es 1972 jeweils zwischen zwölf und 16. Vgl. hierzu StAZH, Z 98, Dossiers Nr. 2415-2485 und 34753547.

123 Vgl. StAZH, Z 98, Dossier Nr. 3483, Gutachten vom 07.06.1972, 8-10, und Krankengeschichte, 4-7.

124 Heinz Stefan Herzka war im erwähnten Gespräch (Anm. 117) gar der Meinung, dass die Tests in der Regel Vorannahmen nicht nur bestätigten, sondern kaum darüberhinausgehende Erkenntnisse brachten, geschweige denn eine bestehende Diagnose umstossen konnten.

125 Herzka 1973, 141.

126 StAZH, Z 98, Dossier Nr. 3483, Krankengeschichte, 6-6r, Eintrag Pat. nach dem gestrigen Besuch von Km und Stv [...], 16.03.1972. 
nen Niederschlag, obwohl das Resultat die Empfehlung des Assistenzarztes stützte.

Die «gute Erziehung» der Pflegemutter zeigte sich bei Roland in seiner Angepasstheit, aber nicht in seiner Entfaltung der Persönlichkeit. Die Persönlichkeitsentwicklung war vielmehr durch die zugleich als «verwöhnend» wie «dominierend, kaptiv und erzieherisch dressierend» charakterisierte Pflegemutter gehemmt worden. Während bei Anna aufgrund des Hin und Hers zwischen Mutter und Grossmutter und der «verwöhnenden» Erziehung eine «frühkindliche Schädigung» angenommen wurde, war eine solche Schädigung bei Roland für den Assistenzarzt aufgrund des «geordneten, harmonischen Familienlebens» bei den Pflegeeltern ausgeschlossen. Dazu beigetragen haben mag, dass die Pflegeltern angaben, Roland habe ihnen nie Erziehungsschwierigkeiten bereitet. Sie machten vielmehr geltend, dass er erst von den regelmässigen Besuchen bei seiner Mutter, welche der Vormund im Hinblick auf die Umplatzierung angeordnet hatte, «verstört» zurückgekommen sei. Wohl deshalb waren die von den Pflegeltern erwähnten und während der ärztlichen Untersuchung beobachteten Verhaltensweisen des Kindes in der ambulanten Begutachtung in Winterthur nicht als «abnorme», sondern als «vorwiegend direkte Reaktionen» auf die beabsichtigte Umplatzierung gedeutet worden. Roland erschien dem dort verantwortlichen Oberarzt, Heinz Stefan Herzka, und der für die Begutachtung zuständigen Assistenzärztin durch die neue Situation stark beunruhigt. Sie gingen aber davon aus, dass die frühkindliche Entwicklung «normal» verlaufen war. ${ }^{127}$

Die in den Gutachten als Symptome des Krankheitsbildes beschriebenen Verhaltensweisen waren für alle Beteiligten anschlussfähig, weil sie alltagssprachliche Begriffe beziehungsweise Adjektive umfassten. ${ }^{128}$ Die von Erich Benjamin in einem Inventar zusammengestellten Verhaltensweisen waren zudem, wie er festhielt, beliebig erweiterbar. ${ }^{129}$ Entscheidend für ihre Bedeutung in der ärztlichen Begutachtung war die Zuordnung zum relativ starren Phasenmodell der kindlichen Persönlichkeitsentwicklung in der damaligen Kinderpsychiatrie. Die in der stationären Begutachtung bei beiden Kindern konstatierten und für das Krankheitsbild typischen «abnormen» Reaktionen wichen nur leicht vom Entwicklungsstand eines Sechsjährigen, wie Roland es war, ab, entsprachen aber nicht dem Entwicklungsstand einer Achtjährigen, wie es Anna zur Zeit ihrer Begutachtung in der Brüschhalde

127 StAZH, Z 98, Dossier Nr. 3483, Ärztlicher Bericht, 8.6.1971, und Zusammenfassung der Gemeinsamen Besprechung, 4.5.1971.

128 Das gilt auch für andere Krankheitsbilder wie die Psychopathie, vgl. Businger/Ramsauer 2019, 86.

129 Benjamin 1938, 200. 
war. Neben dem Alter zeigen sich darüber hinaus geschlechtsspezifische Unterschiede, welche die Störung der Persönlichkeitsentwicklung von Anna als gravierender erscheinen liessen: Das geschilderte aggressive Verhalten und Geltungsstreben wurden bei Roland weniger negativ gewertet als bei Anna. So vermerkte die Kindergärtnerin in ihrem Bericht, dass Roland gelernt habe, sich bei Gleichaltrigen durchzusetzen. ${ }^{130}$ Auf die Erzieherin wirkte der «kleine Wichtigtuer und Gernegross», wie sie zuhanden des Arztes festhielt, «nicht bösartig», sondern «eher drollig». Roland war «überhaupt erzieherisch leicht zu führen» und «seine Schwierigkeiten» muteten die Erzieherin «eher natürlich an, sind eigentlich erstaunlich normal». ${ }^{131}$

Im «Interesse des Kindes» unter psychiatrischer Beobachtung

Rolands leichte Entwicklungshemmung deutete für den begutachtenden Assistenzarzt nicht auf eine «abnorme» Persönlichkeitsentwicklung hin. Trotzdem blieb, wie der Arzt an der gemeinsamen Besprechung festhielt, an der auch der Vormund von Roland anwesend war, die Prognose offen und von den Massnahmen abhängig. Positiv bewertete der Arzt die in der Brüschhalde festgestellte Beruhigung und Lockerung des Knaben, seine gute Intelligenz, seine Anpassungsfähigkeit, sein «natürlicher» Kontakt sowie seine gute Erziehung. Als negativ erachtete er die erstarrte Front zwischen der Pflegemutter und der Mutter. Zudem erschien es ihm fraglich, ob Rolands Mutter eine «echte mütterliche Haltung» entwickeln könne. Eine absehbare Problematik sah er darüber hinaus in der «Geburt jüngerer Konkurrenzgeschwister». ${ }^{132}$

Die Frage des Vormundes war, ob die von ihm beabsichtigte Umplatzierung zur Mutter aus psychiatrischer Sicht unterstützt werden konnte. Der leitende Oberarzt und die Assistenzärztin der Zweigstelle Winterthur hatten 1971 festgehalten, Roland empfinde seine Pflegefamilie deutlich als Familie, wo er hingehöre, es seien aber keine ausreichenden Gründe vorhanden, um der leiblichen Mutter, die jetzt einen geordneten Haushalt führe, das Kind zu verwehren. ${ }^{133}$ Der Assistenzarzt in der Brüschhalde empfahl dem Vormund ein Jahr später, dem Wunsch der Kindsmutter «im Interesse des Kindes» stattzugeben. Dies erschien ihm als geringeres, wenngleich nicht risikoloses Wagnis als der Verbleib in der Pflegefamilie. Die Lockerung und Beruhigung

130 StAZH, Z 98, Dossier Nr. 3483, Gutachten vom 07.06.1972, 11, und Kindergartenbericht über Roland, 08.05.1972.

131 StAZH, Z 98, Dossier Nr. 3483, Heimbericht über Roland, 09.05.1972.

132 StAZH, Z 98, Dossier Nr. 3483, Krankengeschichte, 7r, Eintrag Gemeinsame Untersuchung, 08.05.1972.

133 StAZH, Z 98, Dossier Nr. 3483, Zusammenfassung der Gemeinsamen Besprechung, 4.5.1971. 
von Roland in der Brüschhalde erachtete der Assistenzarzt als Beweis, dass in geeigneter Umgebung eine günstige Weiterentwicklung des Knaben möglich ist. Ein Verbleiben in der Pflegefamilie würde jedoch mit «grosser Wahrscheinlichkeit» zu «grossen Schwierigkeiten» führen, zum einen wegen der «einengenden» und zugleich «verwöhnenden» Erziehung, zum anderen, weil damit Rolands Auseinandersetzung mit der Mutter nur hinausgeschoben würde. Der Wechsel zur Kindsmutter bedeutete für den Assistenzarzt aber auch ein gewisses Risiko, da er, wie er dem Vormund in der gemeinsamen Besprechung erörtert hatte, nichts Endgültiges über deren Fähigkeiten, eine «echte» mütterliche Haltung einzunehmen, aussagen könne. Immerhin stand für ihn fest, dass die Kindsmutter und der Stiefvater Roland «echt» zugetan waren und ihm ein «geordnetes Familienleben» bieten können. Zur Empfehlung des Assistenzarztes trug sicher auch bei, dass der Oberarzt der Zweigstelle Winterthur das Vorhaben des Vormundes bereits unterstützt hatte. Denn abweichende psychiatrische Beurteilungen waren äusserst selten und Widerspruch gegenüber einem Oberarzt nicht vorgesehen. ${ }^{134}$

Die durch die Pflegeeltern gesäten Zweifel blieben indes bestehen, obwohl sie im Gutachten keinen Niederschlag fanden. ${ }^{135}$ Auffallend ist, dass sich die Begutachtenden nicht von den Pflegeeltern hatten vereinnahmen lassen. Nach Ansicht des Pflegevaters hätten sie es in der Hand gehabt, dem Vormund «den Rückhalt zu geben, ein Machtwort zu sagen, dass es in nächster Zeit unmöglich sei, Roland hier wegzunehmen». ${ }^{136}$ Unterstützung erhielt der Pflegevater vom Internisten, der Roland wegen Asthma und Fieber behandelt hatte. Dieser war der Meinung, dass der Wechsel zur Mutter «ein grosses seelisches Trauma» für den Knaben bedeuten würde, nicht nur weil ihn seine Pflegeeltern lieb hätten, sondern weil er vor seiner Mutter Angst habe. Bei der «Vorgeschichte» der Mutter, so seine Überzeugung, «dürfte es wohl am Platze sein, dass sie vorerst einmal über einige Zeit ein entsprechend verantwortliches Leben führt». ${ }^{137} \mathrm{Zu}$ diesem Zeitpunkt war die Mutter bereits zwei Jahre verheiratet. Ab den 1970er Jahren wurden solch autoritäre Anordnungen, wie sie der Internist forderte, generell seltener. ${ }^{138}$

Diese Entwicklung allein mit dem Erstarken der Grundrechte zu erklären, würde allerdings zu kurz greifen. Es zeigt sich zwar, dass 1972 deutlich

134 Vgl. Crueger 1973, 29; Galle 2016, 611.

135 StAZH, Z 98, Dossier Nr. 3483, Krankengeschichte, 7r, Eintrag Gemeinsame Untersuchung, 08.05.1972, und Gutachten vom 07.06.1972, 13.

136 StAZH, Z 98, Dossier Nr. 3483, Bericht des Pflegevaters über die Mutter von Roland, 6.

137 StAZH, Z 98, Dossier Nr. 3483, Schreiben Spezialarzt für innere Krankheiten an Spezialarzt für allgemeine Medizin FMH, 26.01.1972.

138 Vgl. Businger/Ramsauer 2019, 129-135. 
mehr Kinder nach Hause entlassen wurden als $1957 .{ }^{139}$ Wiederholt sprachen die Begutachtenden aber von einer Verlegenheitslösung. Die Rückkehr der Kinder in die eigene Familie entsprach zwar in den untersuchten Fällen dem Wunsch der Eltern, aber keineswegs immer der ärztlichen Empfehlung. Nicht nur Pflegefamilien liessen sich schwer finden, ${ }^{140}$ auch gab es insbesondere in den heilpädagogischen Kinder- und Erziehungsheimen meist lange Wartelisten. Die Empfehlungen der Ärztinnen und Ärzte scheiterten oft am eingeschränkten Angebot. ${ }^{141}$ Zwar wurde eine potentielle Störung der kindlichen Persönlichkeitsentwicklung mit immer mehr Tests immer differenzierter zu erfassen versucht. Die Nachfrage an heilpädagogischen und psychotherapeutischen Behandlungsmöglichkeiten überstieg aber das Angebot, das im ambulanten Bereich noch weitgehend auf die städtischen Zentren beschränkt blieb. ${ }^{142}$ Zudem zeigt der zwischen 1957 und 1972 stark zunehmende Anteil von Kindern unverheirateter Mütter und geschiedener Eltern in der Brüschhalde, dass diese in höherem Masse von stationären Begutachtungen betroffen waren und anschliessend auch häufiger fremdplatziert wurden. ${ }^{143}$ Die bürgerliche Kleinfamilie hatte als Ideal sogar an Bedeutung zugelegt.

Bei Roland erfolgte die Entlassung aus der Brüschhalde zur Mutter nicht ohne deren Einwilligung in eine «intensive Elternberatung» durch den Kinderpsychiatrischen Dienst sowie eine weiterführende Therapie. Der Assistenzarzt empfahl schliesslich dem Vormund auch, die Vormundschaft über Roland aufrecht zu erhalten. Die Mutter von Roland stand trotz ihrer Heirat weiterhin unter kinderpsychiatrischer Beobachtung und behördlicher Kontrolle. Voraussetzung für eine Entlassung des Kindes aus der Brüschhalde zu seinen Eltern war, wie sich im Fall von Roland zeigt, eine «intakte» Ehe mit relativ stereotypen Geschlechterrollen sowie geregelte Einkommens- und Wohnverhältnisse. Das «harmonische, geordnete Familienleben» war aus ärztlicher Sicht die Grundbedingung für eine «normale» Persönlichkeitsentwicklung des Kindes. Daran anknüpfend war für die kindliche Entwicklung die ärztliche Beurteilung der Persönlichkeit von Pflegeeltern und Eltern, insbesondere der Kindsmutter, massgebend.

139 Während 1957 nur 32 Prozent der Kinder in die eigene Familie entlassen wurden, waren es 1972 mit 55 Prozent deutlich mehr.

140 Vgl. Galle 2016, 157.

141 KPD, Jahresbericht 1972, 2.

142 KPD, Jahresbericht 1969, 11-12, und Jahresbericht 1970, 11.

143 Der Anteil unverheirateter Mütter und geschiedener Eltern stieg zwischen 1957 und 1972 von 50 auf 70 Prozent an. Vgl. hierzu StAZH, Z 98, Dossiers 1957 und 1972. Zur Thematik der unehelichen Kinder und der «Scheidungswaisen» vgl. Businger/Ramsauer 2019, 21-29, die bei den Vormundschaftsbehörden ebenfalls eine Zunahme konstatierten. 


\section{Konklusion}

Im Zentrum der behördlich eingeforderten fachärztlichen Begutachtung stand die Persönlichkeit des Kindes. Sie bildete die zentrale Frage der Auftraggeber. Von der Antwort erhofften sich die Vormunde Kenntnisse über die künftige Entwicklung des Kindes und eine Empfehlung zu seiner Platzierung. In beiden von uns untersuchten Fällen legitimierte die psychiatrische Expertise das vom Vormund intendierte Vorgehen, was durchaus der damaligen kinder- und jugendpsychiatrischen Praxis entsprach. ${ }^{144}$ Neben weiteren Gemeinsamkeiten der beiden Begutachtungen gibt es aber auch Anzeichen für einen Wandel, wie die Persönlichkeit von Kindern konzeptualisiert, erfasst und bewertet wurde. Dies wird bereits in den massgeblichen Lehrbüchern deutlich.

Während Erich Benjamin «neurotische» Reaktionen 1938 noch mit Erziehungsschwierigkeiten des Kindes gleichsetzte, fokussierten Moritz Tramer und Jakob Lutz stärker auf die kindliche Entwicklung. Die Perspektive verschob sich von den «Schwierigkeiten», die das Kind machte, zu denen, die das Kind hatte. Für Lutz gab es keine «Neurosen» ohne Entwicklungsirritationen. Abweichungen von dem relativ starren Phasenmodell deuteten folglich auf Störungen der Persönlichkeitsentwicklung hin, die in «abnormen» Reaktionen zum Ausdruck kamen. Die Störung galt als umso gravierender, je stärker sie von den angenommenen altersgemässen und geschlechtsspezifischen Verhaltensmustern abwich. Als Ursachen für die Störungen erschienen, gestützt auf die frühe Bindungsforschung, «frühkindliche Schädigungen» am Wahrscheinlichsten. «Neurotische Reaktionen» wurden, so die Annahme, unter anderem durch «ungünstige Milieueinwirkungen» ausgelöst, waren allerdings laut den Lehrbüchern mehrheitlich konstitutionell bedingt oder zumindest begünstigt. Ob die Ärztinnen und Ärzte stärker auf das Milieu oder die Disposition fokussierten, war davon abhängig, wie sie die Familienverhältnisse und die Persönlichkeit der Eltern einschätzten. Wie spekulativ dieses Unterfangen blieb, zeigt sich in den offenen Prognosen. Letztere eröffneten den Verantwortlichen einen grossen Interpretationsspielraum, der es ihnen stets erlaubte, ein potentielles Scheitern der empfohlenen Massnahmen an der bereits erfolgten, möglicherweise ungenügend erkannten «abnormen» Persönlichkeitsentwicklung des Kindes festzumachen.

Kinder wurden immer weniger wie in der Erwachsenenpsychiatrie bestimmten Charaktertypen zugeordnet. Die Kinderpsychiatrie fokussierte vielmehr auf die Persönlichkeit als Ziel der kindlichen Entwicklung. Die Bewer-

144 Vgl. Künzle et al. 2020 [im Druck]; Businger/Ramsauer 2019, 86; Dietrich-Daum/Ralser 2018, 126-128; Galle 2016, 620. 
tung einer gelingenden «Reifung» orientierte sich an den Entwicklungsphasen, denen spezifische Verhaltensweisen zugeordnet waren. ${ }^{145}$ Die kinderpsychiatrische Begutachtung stellte demnach eine Praktik dar, mit der bestimmt wurde, was (noch oder nicht mehr) als «normal» galt. Sie trug zur Normalisierung von Kindheit bei, indem sie Standards für die Bedingungen eines «gesunden» Aufwachsens setzte. ${ }^{146}$ Ausserehelich geborene Kinder wurden als der korrigierenden Erziehung bedürftige Subjekte konstruiert. ${ }^{147}$ Sie waren im untersuchten Zeitraum häufiger von Begutachtungen in der stationären Kinderpsychiatrie betroffen als Kinder verheirateter Eltern. Das hing mit dem Entwicklungsparadigma zusammen, das der frühen Kindheit und den Anforderungen an die Eltern in dieser Phase besondere Bedeutung beimass.

Die Bewertung der kindlichen Persönlichkeit orientierte sich am Potential des Kindes für eine «normale» Entwicklung. Das Persönlichkeitskonzept der damaligen Zürcher Kinderpsychiatrie, das Jakob Lutz mit seinem Lehrbuch von 1961 grundlegend prägte, hatte aber nicht nur die individuelle Entfaltung, sondern auch die gesellschaftliche Assimilation zum Ziel. ${ }^{148}$ Die Kinderpsychiatrie erachtete es wie die Psychiatrie insgesamt als ihre Aufgabe, die soziale Ordnung zu stabilisieren. ${ }^{149}$ Sie war massgeblich daran beteiligt, das Dispositiv der Kinderfürsorge als normalisierendes Projekt zu erhalten. ${ }^{150}$ Die beobachteten Verhaltensweisen der Kinder waren gemessen am Phasenmodell ein Indikator, ob das Ziel einer gesellschaftsfähigen Persönlichkeit erreicht werden konnte. Voraussetzung für eine «normale» Persönlichkeitsentwicklung des Kindes war, daran hatte sich auch 1972 nichts geändert, ein «geordnetes, harmonisches Familienleben». Während es für die Erfassung der kognitiven Fähigkeiten und charakterlichen Entwicklung der Kinder zahlreiche und in immer höherer Zahl zur Anwendung kommende Testverfahren gab, blieb für die ärztliche Beurteilung der Eltern der persönliche Eindruck entscheidend. Geprägt war der ärztliche Blick von der asymmetrischen Beziehung, die nicht nur durch das Setting gegeben, sondern mehrheitlich auch sozial bedingt war. Besonders deutlich zeigt sich der paternalistische Impetus in der Annahme, dass Eltern zunehmend ihre erzieherischen Instinkte verlören, wie es Jakob Lutz wohl nicht zufällig in Zeiten gesellschaftlicher Umbrüche bekräftigte, so Ende der 1960er Jahre und während der Zürcher Jugendunruhen zu Beginn der 1980er Jahre. ${ }^{151}$

145 Vgl. Lutz 1969b, 37-39.

146 Vgl. Kelle 2013.

147 Vgl. Bechter/Guerrini/Ralser 2014, 135.

148 Herzka 1973, 137-138.

149 Vgl. Tanner 2007b, 275-278.

150 Vgl. Bechter/Guerrini/Ralser 2014, 144.

151 Lutz 1969a, 106; Lutz 1984, 13. Vgl. auch Ralser 2017, 569-576.

Gesnerus 77 (2020) 
Die Taxierung des kindlichen Verhaltens als pathologisch ist massgeblich dem psychiatrischen Begutachtungsverfahren geschuldet, das sich kaum veränderte. Zwar wurden wie erwähnt immer mehr Testverfahren durchgeführt und die Persönlichkeitsmerkmale immer umfassender dokumentiert. Die Tests bestätigten aber auch 1972 nach wie vor hauptsächlich die in der Anamnese gewonnenen Einschätzungen. Ebenso bezogen sich die Einträge in der Krankengeschichte auf vorangehende und zielten folgerichtig, wie von Jakob Lutz gefordert, auf eine bestimmte Diagnose hin - in den beiden untersuchten Fällen auf die damals weitaus häufigste Diagnose, die «neurotischen Reaktionen», die im untersuchten Zeitraum kaum an Konturen gewann. Sie blieb vielmehr ein «Sammeltopf» für «bestimmte psychiatrische Syndrome». ${ }^{152}$ Das zeigt sich nicht nur in den Lehrbüchern, sondern spiegelt sich auch in den Testergebnissen der stationären Begutachtung. Die «neurotischen Reaktionen» lösten die Psychopathie als Residualkategorie ab und waren noch in den 1990er Jahren die meistgestellte Diagnose in der Brüschhalde ${ }^{153}$ Erst ab den 1980er Jahren unterschied die Kinderpsychiatrie ähnlich wie beim Autismuskonzept zunehmend zwischen den im Spektrum der Diagnose liegenden Symptomen, so etwa Angstzuständen, Zwangssyndromen, depressiven Zuständen und weiteren. ${ }^{154}$

Zur Stabilität der kinderpsychiatrischen Begutachtung trug in der Beobachtungsstation Brüschhalde die personelle Kontinuität in der Leitung bei. Aufgrund ihrer hohen Arbeitsbelastung waren die Direktoren und die Oberärztinnen und -ärzte, welche die Gutachten autorisierten, indes wenig vor Ort präsent. Das hatte zur Folge, dass die Assistenzärztinnen und -ärzte stark auf Lehrbuchwissen angewiesen waren und ihnen ihre persönliche Lebenserfahrung als Referenz dienen musste.

Die beiden Gutachten weisen trotz standardisierter Form und lehrbuchmässiger Herleitung der Diagnose nicht nur in Sprache und Wertung erhebliche Unterschiede auf, sondern auch in der Begründung der empfohlenen Behandlung und Platzierung. So ist bemerkenswert, dass der Assistenzarzt 1972 die Interessen des Kindes höher gewichtete als die elterlichen Rechte und den gesellschaftlichen Nutzen. Dies entsprach einem Trend in der Rechtsprechung, wie er sich vier Jahre später mit dem Kindeswohl als oberster Rechtsmaxime im Gesetz niederschlug. Im Vergleich zu seiner Kollegin 1957 hatte der Assistenzarzt durch die erweiterte personelle Besetzung aber auch mehr Ressourcen, um Informationen einzuholen, Aussagen einzuordnen und Auswertungen zu verschriftlichen. Doch weder die Ansätze der gesellschaftlichen Li-

152 Canziani 1995, 68.

153 Canziani 1995, 8-9.

154 Canziani 1995, 63. 
beralisierung noch der institutionelle Wandel in der Kinderpsychiatrie jener Jahre vermochte die Situation benachteiligter Familien, insbesondere von Kindern und Frauen, aus heutiger Sicht grundlegend zu verbessern. Die rechtliche und damit verbundene soziale Diskriminierung ausserehelicher Kinder und ihrer Mütter wurde erst 1976 mit der Revision des Familienrechts behoben. Dass die Begutachtenden 1972 deutlich häufiger als noch 1957 eine Rückkehr in die Herkunftsfamilie empfahlen, stellte oft eine Verlegenheitslösung dar. Die Empfehlung steht aber auch im Zusammenhang mit dem erweiterten und differenzierteren ambulanten Therapie- und Beratungsangebot, das sowohl eine spezifische Behandlung psychisch erkrankter Kinder als auch eine fortgesetzte Überwachung der familiären Situation und der elterlichen Obhut ermöglichte. Von der psychiatrischen wie von der behördlichen Kontrolle waren Mütter stärker betroffen als Väter und sahen sich entsprechend häufiger mit Verhaltensnormen konfrontiert. ${ }^{155}$ Massgebend für eine Fremdplatzierung, so lässt sich festhalten, war nicht die Diagnose, die bei den meisten Kindern zunehmend gleich lautete, sondern vielmehr die familiäre Konstellation und die Persönlichkeit der Eltern, allen voran diejenige der Mütter.

\section{Quellen und Literatur}

Ungedruckte Quellen

StAZH, MM 3.123 RRB 1968/2933, Kinderpsychiatrischer Dienst (Personal), 25.07.1968, 1458-1459.

StAZH, Z 98, Dossier Nr. 2422 (1957) [Anna].

StAZH, Z 98, Dossier Nr. 3483 (1972) [Roland].

Gedruckte Quellen

Kinderpsychiatrischer Dienst des Kantons Zürich, Jahresberichte.

\section{Literatur}

Akermann, Martina/Markus Furrer/Sabine Jenzer, Bericht Kinderheime im Kanton Luzern im Zeitraum von 1930-1970 (Luzern 2012).

Ammann, Ruth/Alfred Schwendener, «Zwangslagenleben». Biografien von ehemals administrativ versorgten Menschen, hrsg. v. d. Unabhängigen Expertenkommission (UEK) Administrative Versorgungen (Zürich 2019).

155 Businger/Ramsauer 2019, 14.

Gesnerus 77 (2020) 
Bechter, Anneliese/Flavia Guerrini/Michaela Ralser, Das proletarische Kind und seine uneheliche Mutter als Objekte öffentlicher Erziehung. Zum Fürsorgeregime im Tirol der 1960er- und beginnenden 1970er-Jahre, in: Maria Andrea Wolf/Elisabeth Dietrich-Daum/Eva Fleischer/Maria Heidegger (Hrsg.), Child Care. Kulturen, Konzepte und Politiken der Fremdbetreuung von Kindern (Weinheim 2013) 132-146.

Benjamin, Erich/Heinrich Hanselmann/Max Isserlin/Jakob Lutz/Alexander Ronald, Lehrbuch der Psychopathologie des Kindesalters für Ärzte und Erzieher (ErlenbachZürich/Leipzig 1938).

Benjamin, Erich, Psychopathie und Neurose, in: Erich Benjamin/Heinrich Hanselmann/Max Isserlin/Jakob Lutz/Alexander Ronald, Lehrbuch der Psychopathologie des Kindesalters für Ärzte und Erzieher (Erlenbach-Zürich/Leipzig 1938) 151-239.

Bernet, Brigitta, Assoziationsstörungen. Zum Wechselverhältnis von Krankheitsund Gesellschaftsdeutung im Werk Eugen Bleulers, in: Heiner Fangerau/Karen Nolte (Hrsg.), «Moderne» Anstaltspsychiatrie im 19. und 20. Jahrhundert-Legitimation und Kritik (Stuttgart 2006) 169-193.

Bombach, Clara/Thomas Gabriel/Samuel Keller/Nadja Ramsauer/Alessandra Staiger Marx, Zusammen alleine. Alltag in Winterthurer Kinder- und Jugendheimen 1950-1990 (Winterthur 2017).

Brauchli, Andreas, Das Kindeswohl als Maxime des Rechts (Zürich 1982).

Bühler, Rahel/Sara Galle/Flavia Grossmann/Matthieu Lavoyer/Michael Mülli/ Emmanuel Neuhaus/Nadja Ramsauer, Ordnung, Moral und Zwang. Administrative Versorgungen und Behördenpraxis, hrsg. v. d. Unabhängigen Expertenkommission (UEK) Administrative Versorgungen (Zürich 2019).

Businger, Susanne/Nadja Ramsauer, "Genügend goldene Freiheit gehabt»: Heimplatzierungen von Kindern und Jugendlichen im Kanton Zürich, 1950-1990 (Zürich 2019).

Canziani, Andreas Peter, Veränderungen der Klientel in drei Jahrzehnten stationärer Kinderpsychiatrie. Ein Vergleich von Patienten der Geburtsjahrgänge 1952, 1962 und 1972 aus dem Kinderheim Brüschhalde in Männedorf (Zürich 1995).

Corboz, Jules Robert, Möglichkeiten und Aufgaben der Kinderpsychiatrie in der Erziehungsberatung, in: Praxis der Kinderpsychologie und Kinderpsychiatrie. Zeitschrift für analytische Kinderpsychologie, Psychotherapie und Psychagogik in Praxis und Forschung 18 (1969) 178-183.

Crueger, Manfred, Ärztliches Selbstverständnis im Wandel (Bonn 1973).

Dietrich-Daum, Elisabeth/Michaela Ralser, Kinder zwischen Psychiatrie und Fürsorgeerziehung. Das Beispiel der Innsbrucker Kinderbeobachtungsstation 1954 bis 1987, in: Elisabeth Dietrich-Daum/Michaela Ralser/Elisabeth Lobenwein (Hrsg.), Schwerpunkt: Medikalisierte Kindheiten. Die neue Sorge um das Kind vom ausgehenden 19. bis ins späte 20. Jahrhundert, in: Virus. Beiträge zur Sozialgeschichte der Medizin 17 (Leipzig 2018) 111-130.

Dietrich-Daum, Elisabeth/Michaela Ralser/Dirk Rupnow, Psychiatrisierte Kindheiten. Die Innsbrucker Kinderbeobachtungsstation Maria Nowak-Vogl (Innsbruck 2019).

Dubach, Roswitha, Verhütungspolitik. Sterilisationen im Spannungsfeld von Psychiatrie, Gesellschaft und individuellen Interessen in Zürich (1890-1970) (Zürich 2013). 
Egger, August, Die Verwandtschaft. Art. 252-359 ZGB. Kommentar zum Schweizerischen Zivilgesetzbuch, Bd. 2: Das Familienrecht, 2. Abteilung (Zürich 21943).

Furrer, Markus/Kevin Heiniger/Thomas Huonker/Sabine Jenzer/Anne-Françoise Praz (Hrsg.), Fürsorge und Zwang. Fremdplatzierung von Kindern und Jugendlichen in der Schweiz 1850-1980, Itinera 36 (Basel 2014).

Galle, Sara, Kindswegnahmen. Das «Hilfswerk für die Kinder der Landstrasse» der Stiftung Pro Juventute im Kontext der schweizerischen Jugendfürsorge (Zürich 2016).

Geisthövel, Alexa/Volker Hess, Handelndes Wissen. Die Praxis des Gutachtens, in: dies. (Hrsg.), Medizinisches Gutachten. Geschichte einer neuzeitlichen Praxis (Göttingen 2017) 9-39.

Germann, Urs, Plausible Geschichten. Zur narrativen Qualität gerichtspsychiatrischer Gutachten um 1900, in: Alexa Geisthövel/Volker Hess (Hrsg.), Medizinisches Gutachten. Geschichte einer neuzeitlichen Praxis (Göttingen 2017) 318-339.

Germann, Urs, Psychiatrie und Strafjustiz. Entstehung, Praxis und Ausdifferenzierung der forensischen Psychiatrie in der deutschsprachigen Schweiz 1850-1950 (Zürich 2004).

Germann, Urs, Vom Klecks zum Psychogramm. Der Rorschach-Formdeutversuch als Aufführungs- und Aufzeichnungsverfahren, in: Monika Ankele/Céline Kaiser/ Sophie Ledebur (Hrsg.), Aufführen - Aufzeichnen - Anordnen. Wissenspraktiken in Psychiatrie und Psychotherapie (Wiesbaden 2019) 123-145.

Goffman, Erving, Asyle. Über die soziale Situation psychiatrischer Patienten und anderer Insassen (Frankfurt a. M. 1973).

Hähner-Rombach, Sylvelyn, Patientinnen und Patienten der Kinderbeobachtungsstation Innsbruck. Einweisung und Aufenthalt zwischen 1949 und 1989 im Spiegel der Krankenakten, in: Medizinhistorisches Journal 52/4 (2017) 308-351.

Hauss, Gisela/Thomas Gabriel/Martin Lengwiler (Hrsg.), Fremdplatziert. Heimerziehung in der Schweiz, 1940-1990 (Zürich 2018).

Hauss, Gisela/Béatrice Ziegler (Hrsg.), Helfen, Erziehen, Verwalten. Beiträge zur Geschichte der Sozialen Arbeit in St. Gallen (Zürich 2009).

Heese, Gerhard, Hanselmann, Heinrich, in: HLS online, Version vom 16.03.2017, URL: https://hls-dhs-dss.ch/de/articles/009029/2017-03-16/.

Herzka, Heinz Stefan, Der rechtliche Kinderschutz aus kinderpsychiatrischer Sicht, in: Zeitschrift für Vormundschaftswesen 28/4 (1973) 136-143.

Herzka, Heinz Stefan, Kinderpsychiatrische Krankheitsbilder. Kasuistisches Lehrbuch mit Testübersicht und Bibliographie für ärztliche, psychologische, pädagogische und soziale Berufe (Basel 1978).

Hiltmann, Hildegard, Kompendium der psychodiagnostischen Tests (Bern/Stuttgart 1960).

Hoffmann, Sven Olaf, Psychoneurosen und Charakterneurosen, in: Karl Peter Kisker/ Hans Lauter/Joachim-Ernst Meyer/Christian Müller/Erik Strömgren (Hrsg.), Neurosen, psychosomatische Erkrankungen, Psychotherapie, in: Psychiatrie der Gegenwart 1 (Berlin ${ }^{3} 1986$ ) 29-62.

Imboden, Gabriela/Hans Jakob Ritter, «... jedenfalls ist Petentin Trägerin einer denkbar ungünstigen Erbmasse». Eugenik in Psychiatrie- und Verwaltungsakten, in: Claudia Kaufmann/Walter Leimgruber (Hrsg.), Was Akten bewirken können. Integrations- und Ausschlussprozesse eines Verwaltungsvorgangs (Zürich 2008) 92-103. 
Janett, Mirjam, Die behördliche «Sorge» um das Kind. Psychiatrische Konzepte und fürsorgerische Massnahmen in Basel-Stadt (1945-1972), in: Elisabeth DietrichDaum/Michaela Ralser/Elisabeth Lobenwein (Hrsg.), Schwerpunkt: Medikalisierte Kindheiten. Die neue Sorge um das Kind vom ausgehenden 19. bis ins späte 20. Jahrhundert, Virus. Beiträge zur Sozialgeschichte der Medizin 17 (Leipzig 2018) 257-265.

Jenzer, Sabine/Willi Keller/Thomas Meier, Eingeschlossen. Alltag und Aufbruch in der psychiatrischen Klinik Burghölzli zur Zeit der Brandkatastrophe von 1971 (Zürich 2017).

Jenzer, Sabine/Thomas Meier, Die Zürcher Anstaltslandschaft 1876-2017, in: Beat Gnädinger/Verena Rothenbühler (Hrsg.), Menschen korrigieren. Fürsorgerische Zwangsmassnahmen und Fremdplatzierungen im Kanton Zürich bis 1981 (Zürich 2018) 75-145.

Jorisch-Wissink, Ellen, Der Kinderpsychiater Moritz Tramer (1882-1963), Zürcher medizingeschichtliche Abhandlungen 184 (Zürich 1986).

Kelle, Helga, Normierung und Normalisierung der Kindheit. Zur (Un)Unterscheidbarkeit und Bestimmung der Begriffe, in: Helga Kelle/Johanna Mierendorff (Hrsg.), Normierung und Normalisierung der Kindheit (Weinheim 2013) 15-37.

Kinderpsychiatrischer Dienst des Kantons Zürich, Jahresberichte 1957-1972.

Kochmann, Rudolf, Über Diagnose und Prognose, besonders der Psychopathie, in der Kinderpsychiatrie, in: Acta Paedopsychiatrica 30 (1963) 21-28.

Künzle, Lena/Daniel Lis/Sara Galle/Emmanuel Neuhaus/Iris Ritzmann, Legitimierung behördlicher Praxis? Analyse einer stationären kinderpsychiatrischen Begutachtung in Zürich im Jahr 1944, in: Österreichische Zeitschrift für Geschichtswissenschaften OeZG 31/2 (2020) [im Druck].

Lengwiler, Martin/Gisela Hauss/Thomas Gabriel/Anne-Françoise Praz/Urs Germann, Bestandsaufnahme der bestehenden Forschungsprojekte in Sachen Verding- und Heimkinder. Bericht zuhanden des Bundesamts für Justiz EJPD (Basel 2013).

Lengwiler, Martin, Standardisierung oder Individualdiagnostik? Ärztliche Gutachten im Versicherungswesen des 20. Jahrhunderts, in: Alexa Geisthövel/Volker Hess (Hrsg.), Medizinisches Gutachten. Geschichte einer neuzeitlichen Praxis (Göttingen 2017) 95-120.

Lutz, Jakob, Das kantonale Kinderhaus Stephansburg Zürich 8, in: Jahresbericht des Zürcher Hülfsvereins für Geisteskranke 1929 (Zürich 1929).

Lutz, Jakob, Der Beitrag der Kinderpsychiatrie für die Jugendhilfe im Kanton Zürich, in: Festschrift zum 50jährigen Bestehen des kantonalen Jugendamtes (Zürich 1969a) 101-107.

Lutz, Jakob, Gesunde und abnorme Bahnen der Beziehungsentwicklung zwischen Kind und Eltern, in: Das schwer erziehbare Kind. 7. Kongress vom 18.-20. Juni 1968 in Köln, hrsg. v. Landesverband Rheinland - Landesjugendamt (1969b) 36-45.

Lutz, Jakob, Kinderpsychiatrie. Eine Anleitung zu Studium und Praxis für Ärzte, Erzieher, Fürsorger, Richter. Mit besonderer Berücksichtigung heilpädagogischer Probleme (Zürich/Stuttgart 1961).

Lutz, Jakob, Kinderpsychiatrie. Eine Anleitung zu Studium und Praxis für Ärzte, Erzieher, Fürsorger, Richter. Mit besonderer Berücksichtigung heilpädagogischer Probleme (Zürich ${ }^{4} 1972$ ).

Lutz, Jakob, Krankhafte psychische Entwicklungen im Kindes- und Jugendlichenalter, in: Universitas. Zeitschrift für Wissenschaft, Kunst und Kultur 22/1 (1967) 67-79. 
Lutz, Jakob, Über die psychiatrische Beobachtung bei Kindern und Jugendlichen im Kanton Zürich (Zürich 1935).

Lutz, Jakob, Verwandlungen im Menschenleben. Ganzheit in den Lebensstufen von Kindheit und Jugend, Reife und Alter, in: Schweizer Heimwesen. Fachblatt VSA 55/1 (1984) 8-15.

Lutz, Jakob, Zur Frage der Mütterlichkeit (Bemerkungen zu Bowlbys Buch «Soins maternels et santé mentale», 1954), in: Acta Paedopsychiatrica 24 (1957) 13-18.

Meier, Marietta, Die Konstruktion von Wissen durch Fallgeschichten. Psychochirurgische Studien in den 1940er und 1950er Jahren, in: Arne Höcker/Jeannine Moser/ Philippe Weber (Hrsg.), Wissen. Erzählen. Narrative der Humanwissenschaften (Bielefeld 2006) 103-114.

Meier, Marietta, Progredienter Verlauf. Koordinaten einer Krankengeschichte, in: Cornelius Borck/Armin Schäfer (Hrsg.), Das psychiatrische Aufschreibesystem (Paderborn 2015) 245-263.

Meier, Marietta/Urs Germann/Roswitha Dubach/Brigitta Bernet, Zwang zur Ordnung. Psychiatrie im Kanton Zürich, 1870-1970 (Zürich 2007).

Meier, Marietta, «Soziale Heilung» als Ziel psychochirurgischer Eingriffe. Leukotomie im Spannungsfeld von Individuum, Anstalt und Gesellschaft, in: Schweizerische Zeitschrift für Geschichte 4 (2004) 410-425.

Oechsle, Susanne, Erich Benjamin (1880-1943). Leben und Werk eines jüdischen Wissenschaftlers und Kinderarztes (Hamburg 2006).

Ralser, Michaela, Die Sorge um das erziehungsschwierige Kind, in: Heiner Fange$\mathrm{rau} /$ Sascha Tschopp/Klaus Schepker (Hrsg.), Kinder- und Jugendpsychiatrie im Nationalsozialismus und in der Nachkriegszeit. Zur Geschichte ihrer Konsolidierung (Berlin 2017) 557-578.

Ramsauer, Nadja, «Verwahrlost». Kindswegnahmen und die Entstehung der Jugendfürsorge im schweizerischen Sozialstaat 1900-1945 (Zürich 2000).

Ries, Markus/Valentin Beck, Hinter Mauern. Fürsorge und Gewalt in kirchlich geführten Erziehungsanstalten im Kanton Luzern (Zürich 2013).

Steinhausen, Hans-Christoph, Lutz, Jakob, in: HLS online, Version vom 03.07.2008, URL: https://hls-dhs-dss.ch/de/articles/014459/2008-07-03/.

Tanner, Jakob, Eugenik und Rassenhygiene in Wissenschaft und Politik seit dem ausgehenden 19. Jahrhundert. Ein historischer Überblick, in: Michael Zimmermann (Hrsg.), Zwischen Erziehung und Vernichtung. Zigeunerpolitik und Zigeunerforschung im Europa des 20. Jahrhunderts, Beiträge zur Geschichte der Deutschen Forschungsgemeinschaft 3 (Stuttgart 2007a) 109-121.

Tanner, Jakob, Ordnungsstörungen. Konjunkturen und Zäsuren in der Geschichte der Psychiatrie, in: Marietta Meier/Brigitta Bernet/Roswitha Dubach/Urs Germann (Hrsg.), Zwang zur Ordnung. Psychiatrie im Kanton Zürich, 1870-1970 (Zürich 2007b) 271-306.

Tramer, Moritz, Lehrbuch der allgemeinen Kinderpsychiatrie. Einschliesslich der allgemeinen Psychiatrie der Pubertät und Adoleszenz (Basel $\left.{ }^{3} 1949\right)$.

Tuor, Peter, Das schweizerische Zivilgesetzbuch. Eine systematische Darstellung mit Berücksichtigung der Rechtsprechung des Schweizerischen Bundesgerichts (Zürich ${ }^{81968) .}$

Zürrer-Simmen, Susanna, Wege zu einer Kinderpsychiatrie in Zürich, Zürcher medizingeschichtliche Abhandlungen 257 (Dietikon 1994). 